\title{
Synthesis and Biological Evaluation of Purealin and Analogues as Cytoplasmic Dynein Heavy Chain Inhibitors
}

\author{
Guangyu Zhu, Fanglong Yang, Raghavan Balachandran, Peter Höök, \\ Richard B. Vallee, Dennis P. Curran and Billy W. Day*
}

\section{Supporting Information}

Representative HPLC chromatograms for key compounds subjected to biological testing:

Compound

Page

1 (purealin) -1

1 (purealin) -2

S3

2 (lipopurealin A)

S4

3 (lipopurealin B)

S5

4 (lipopurealin C)

S6

5 (purealidin A) -1

S7

5 (purealidin A) -2

S8

5 (purealidin A) -3

S9

$40 \mathrm{a}$

S10

$40 \mathrm{~b}$

$\mathrm{S} 11$

$40 \mathrm{c}$

S12

$40 \mathrm{~d}$

$\mathrm{S} 13$

$41 \mathrm{a}$

S14

$41 \mathrm{~b}$

S15

$41 \mathrm{c}-1$

S16

$41 \mathrm{c}-2$

S17

$41 d$

S18

$42 \mathrm{a}$

S19

$42 \mathrm{~b}$

S20

$42 \mathrm{c}$

S21

$42 \mathrm{~d}$

S22

$43 \mathrm{a}$

S23

$43 b$

S24

$43 \mathrm{c}$

S25

$43 d$

S26 


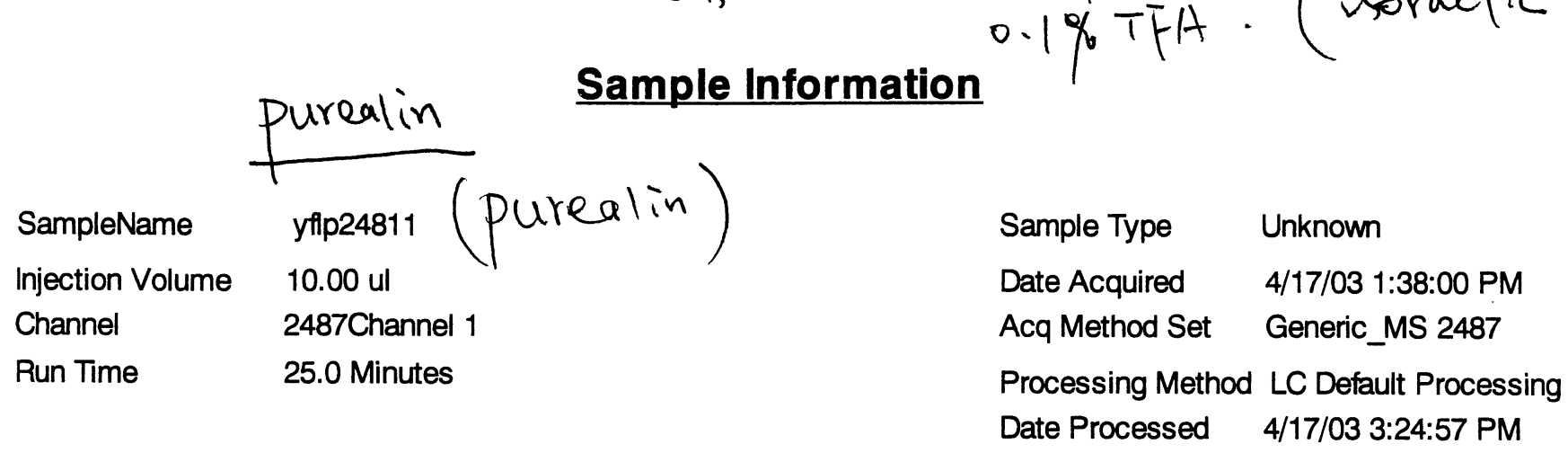

sample_info Enter Comment here!

Auto-Scaled Chromatogram

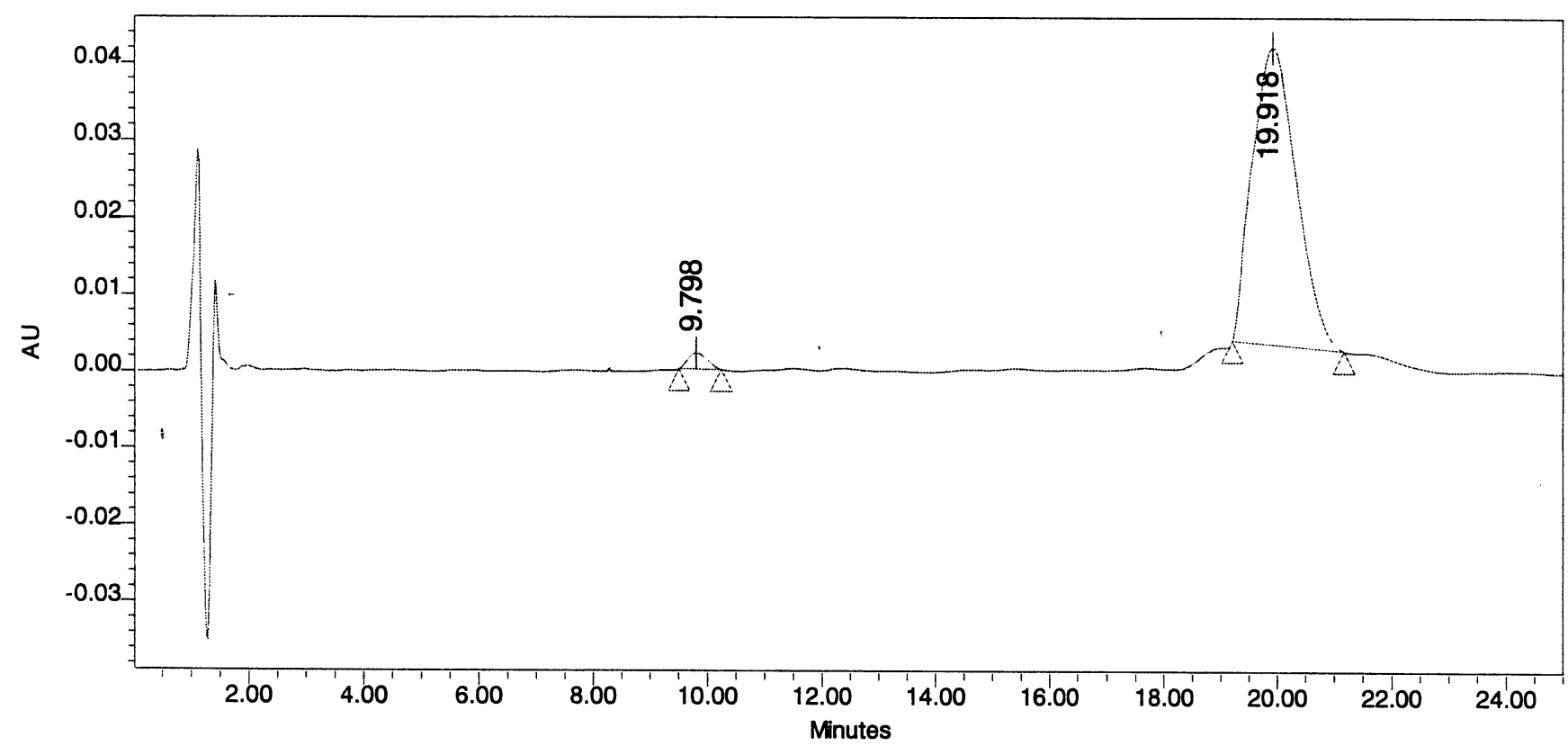

Peak Results

\begin{tabular}{|r|r|r|r|r|l|}
\hline & Name & RT & Area & \% Area & Peak Type \\
\hline 1 & & 9.798 & 47628 & 2.23 & Unknow n \\
\hline 2 & & 19.918 & 2088252 & 97.77 & Unknow n \\
\hline
\end{tabular}




\section{Sample Information}

\begin{tabular}{|c|c|c|c|}
\hline SampleName & yfl-purealin-8 & Sample Type & Unknow n \\
\hline Vial & 1 & Date Acquired & 11/1/2005 10:47:27 AM \\
\hline Injection & 1 & Acq Method Set & fanglong \\
\hline Injection Volume & $10.00 \mathrm{ul}$ & Processing Methoc & d LC Default Processing \\
\hline Channel & 2487Channel 2 & Date Processed & 11/4/2005 3:23:02 PM \\
\hline Run Time & 40.0 Minutes & & \\
\hline
\end{tabular}

\section{Auto-Scaled Chromatogram}

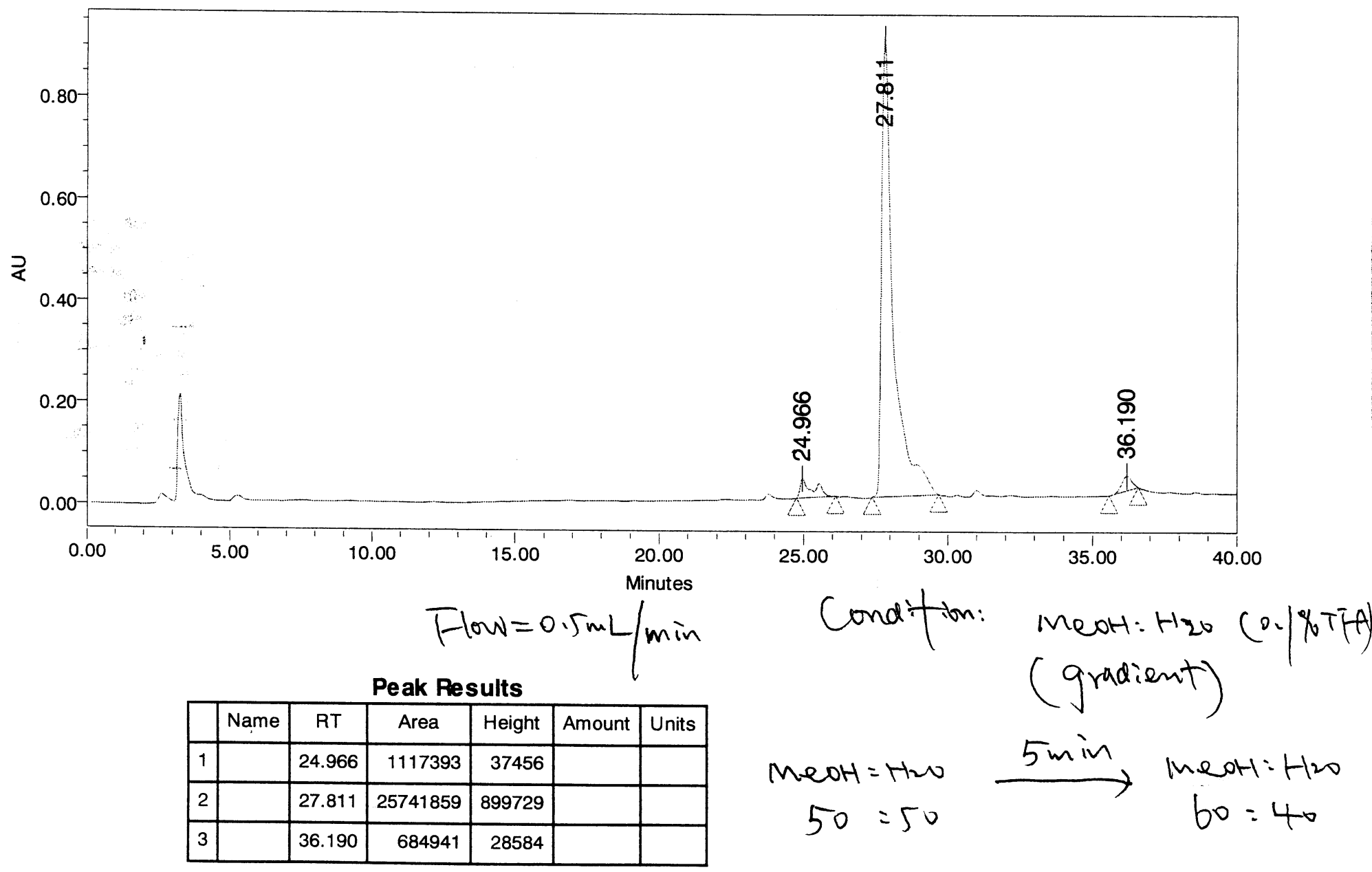




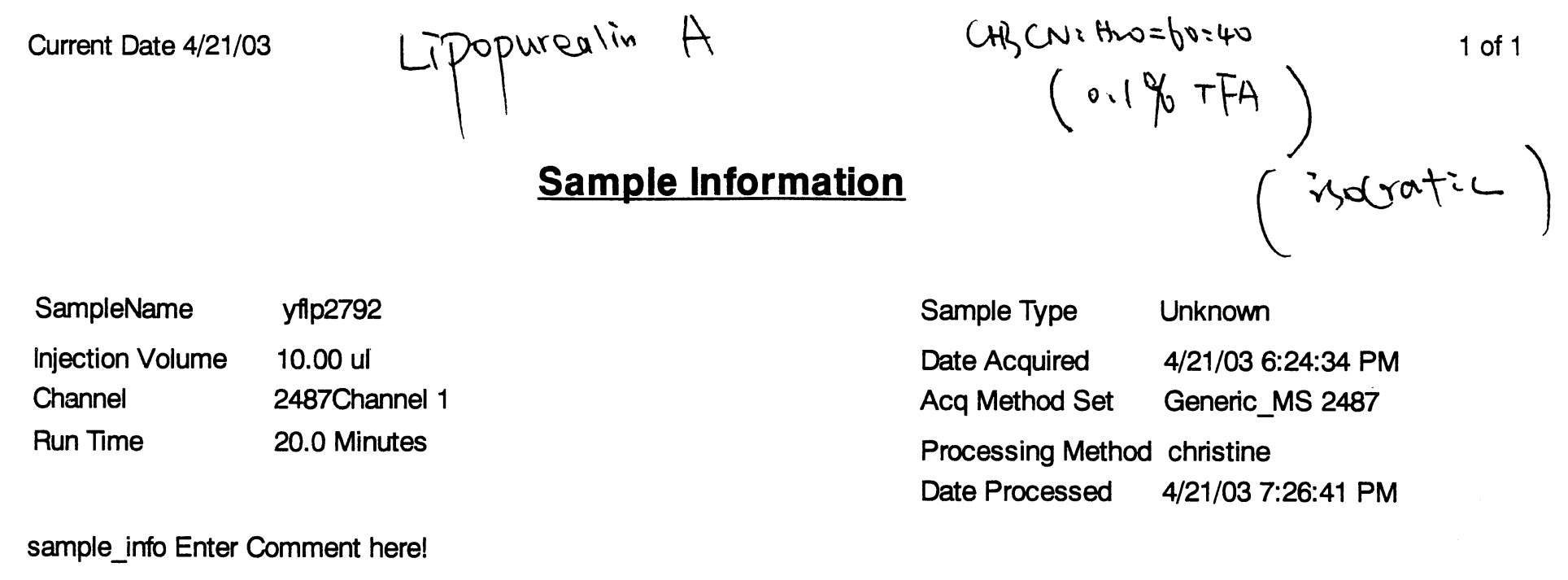

Auto-Scaled Chromatogram

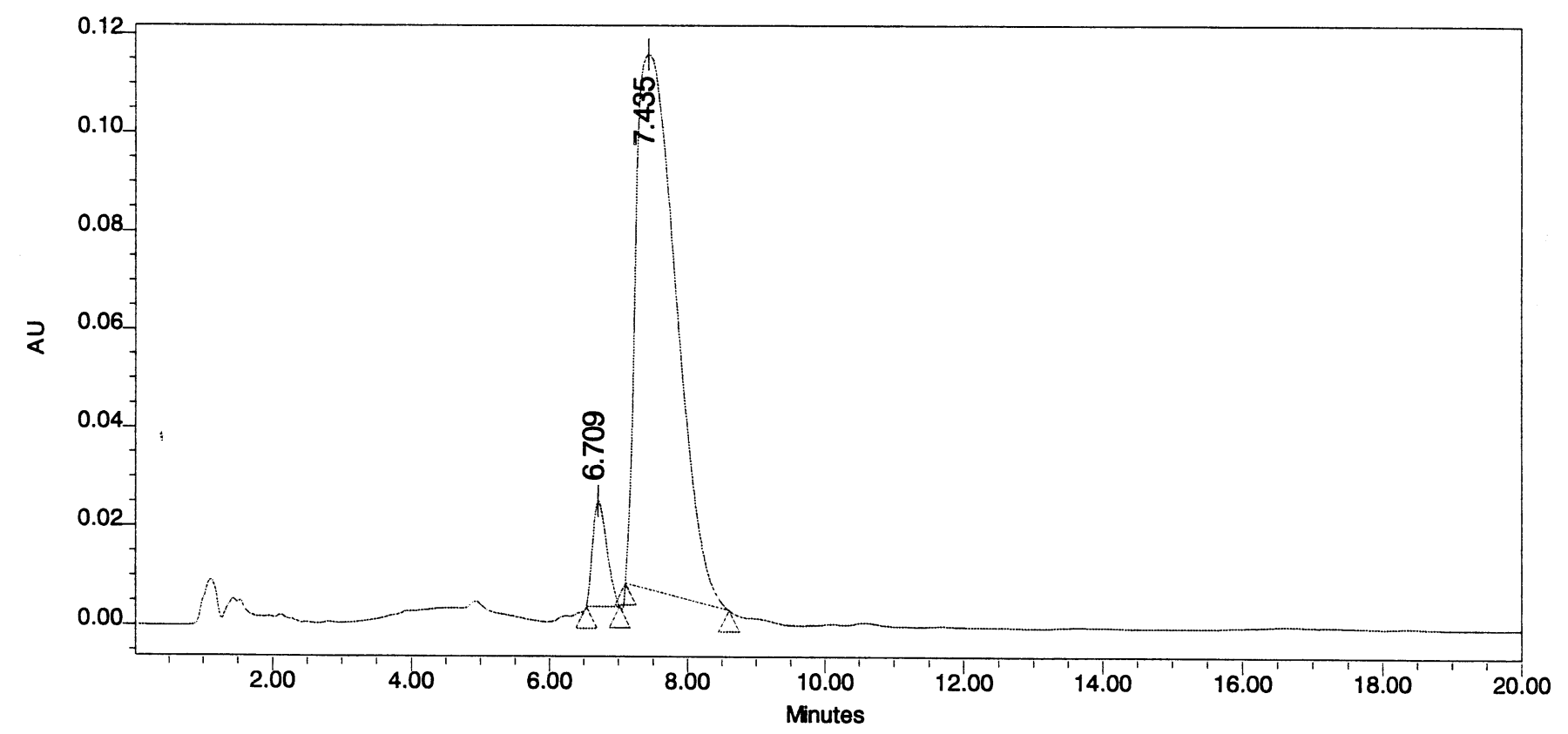

Peak Results

\begin{tabular}{|r|c|c|c|r|l|}
\hline & Name & RT & Area & \% Area & Peak Type \\
\hline 1 & & $6: 709$ & 301021 & 6.44 & Unknow n \\
\hline 2 & & 7.435 & 4372316 & 93.56 & Unknow $n$ \\
\hline
\end{tabular}




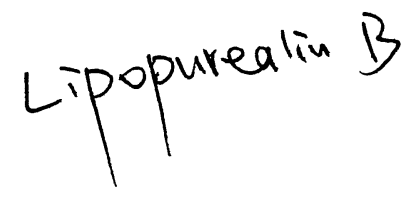

Sample Information

$$
\begin{gathered}
C H 3 C N=H_{20}=60: 40 \\
(0.1 \%-F A)
\end{gathered}
$$

$\begin{array}{ll}\text { SampleName } & \text { yflp2771 } \\ \text { Injection Volume } & 10.00 \mathrm{ul} \\ \text { Channel } & \text { 2487Channel 1 } \\ \text { Run Time } & \text { 30.0 Minutes }\end{array}$

sample_info Enter Comment here!
Sample Type Unknown

Date Acquired $\quad$ 4/21/03 5:31:09 PM

Acq Method Set Generic_MS 2487

Processing Method christine

Date Processed 4/21/03 7:20:36 PM

\section{Auto-Scaled Chromatogram}

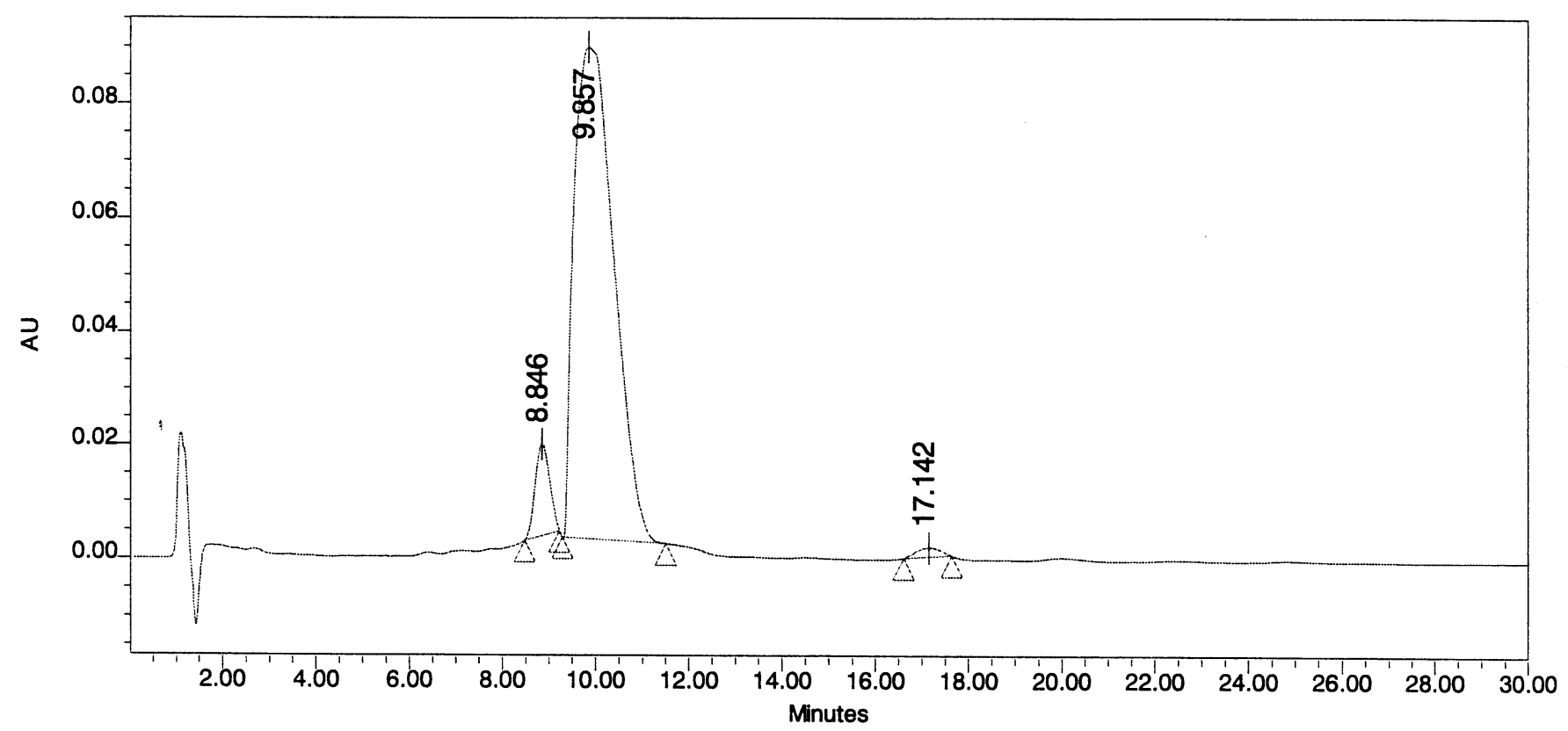

\section{Peak Results}

\begin{tabular}{|r|r|r|r|r|l|}
\hline & Name & RT & Area & \% Area & Peak Type \\
\hline 1 & & 8.846 & 352036 & 6.37 & Unknow n \\
\hline 2 & & 9.857 & 5111976 & 92.56 & Unknow n \\
\hline 3 & & 17.142 & 59019 & 1.07 & Unknow $\mathrm{n}$ \\
\hline
\end{tabular}


Current Date 4/17/03

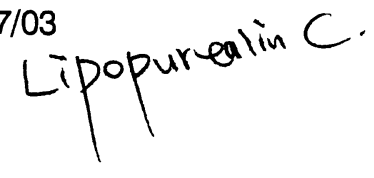

$$
C H \beta C N=H_{20}=60=40 \quad \otimes(0.1 \% 0 T A)
$$

$$
\text { Ahalytic (ivocratic) }
$$

\section{Sample Information}

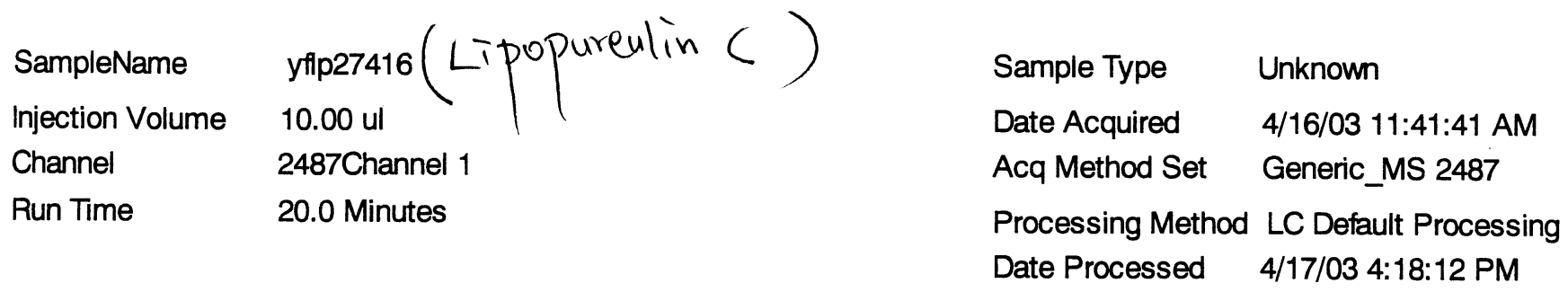

sample_info Enter Comment here!

Auto-Scaled Chromatogram

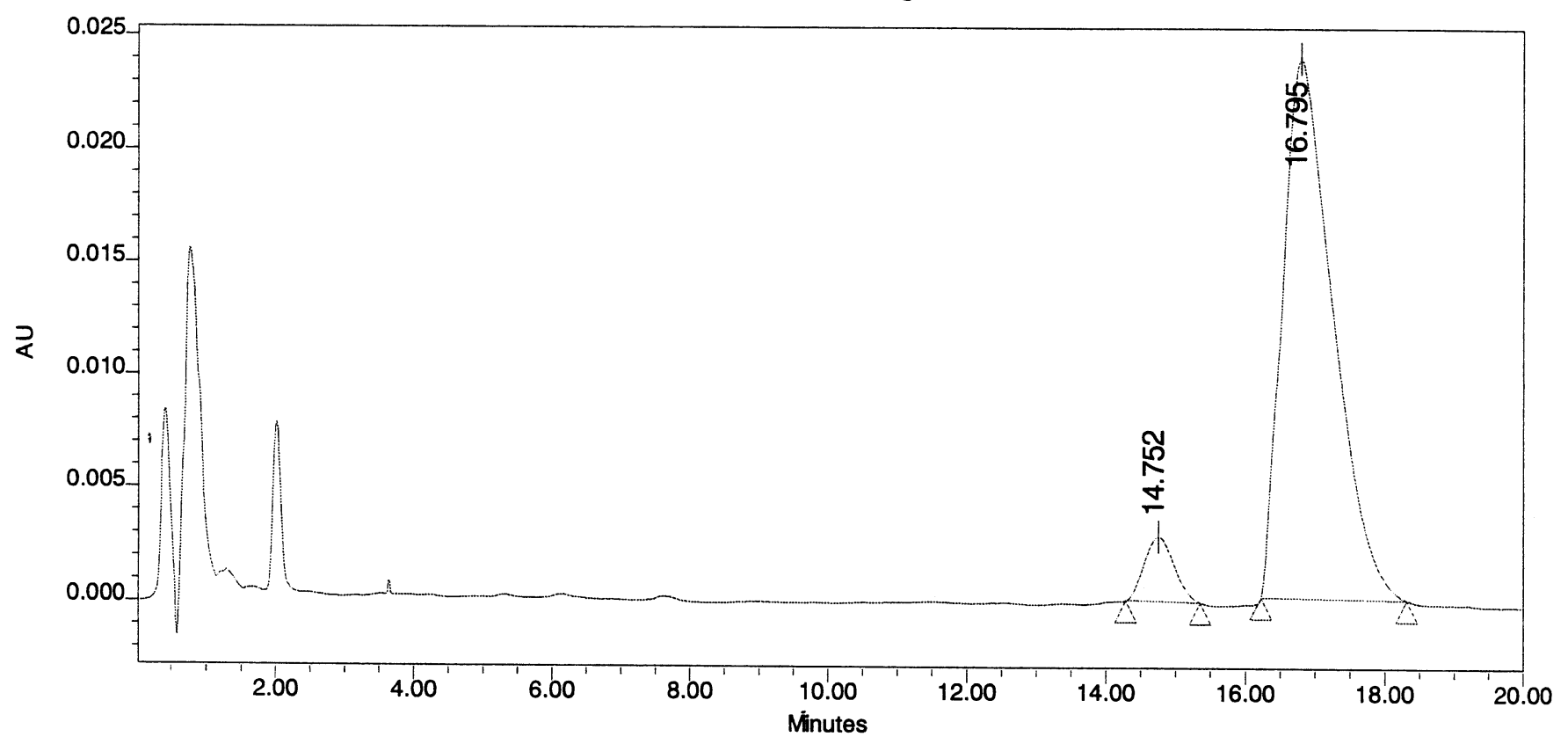

Peak Results

\begin{tabular}{|l|r|r|r|r|l|}
\hline & Name & RT & \multicolumn{1}{c|}{ Area } & $\%$ Area & Peak Type \\
\hline 1 & & 14.752 & 83309 & 6.53 & Unknow $n$ \\
\hline 2 & & 16.795 & 1191666 & 93.47 & Unknow $n$ \\
\hline
\end{tabular}


HU. HuNm

Sample Information

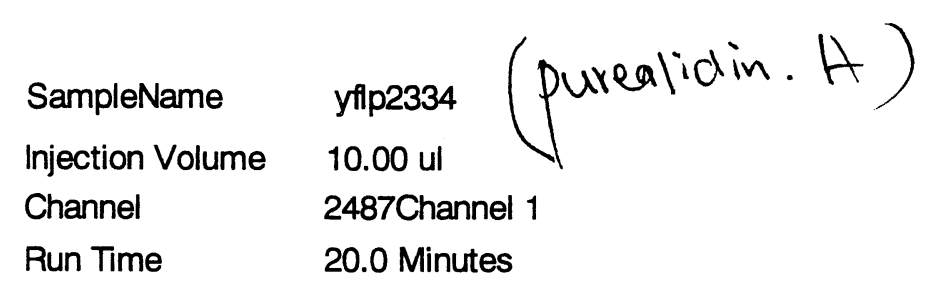

$C H$ CN $/ H_{20}=15: 85^{1}$ of 1 (0.1\%TFA.)

zsocratic

Sample Type Unknown

Date Acquired $\quad$ 4/21/03 9:50:49 PM

Acq Method Set Generic_MS 2487

Processing Method christine

Date Processed $\quad$ 4/21/03 10:13:26 PM

sample_info Enter Comment here!

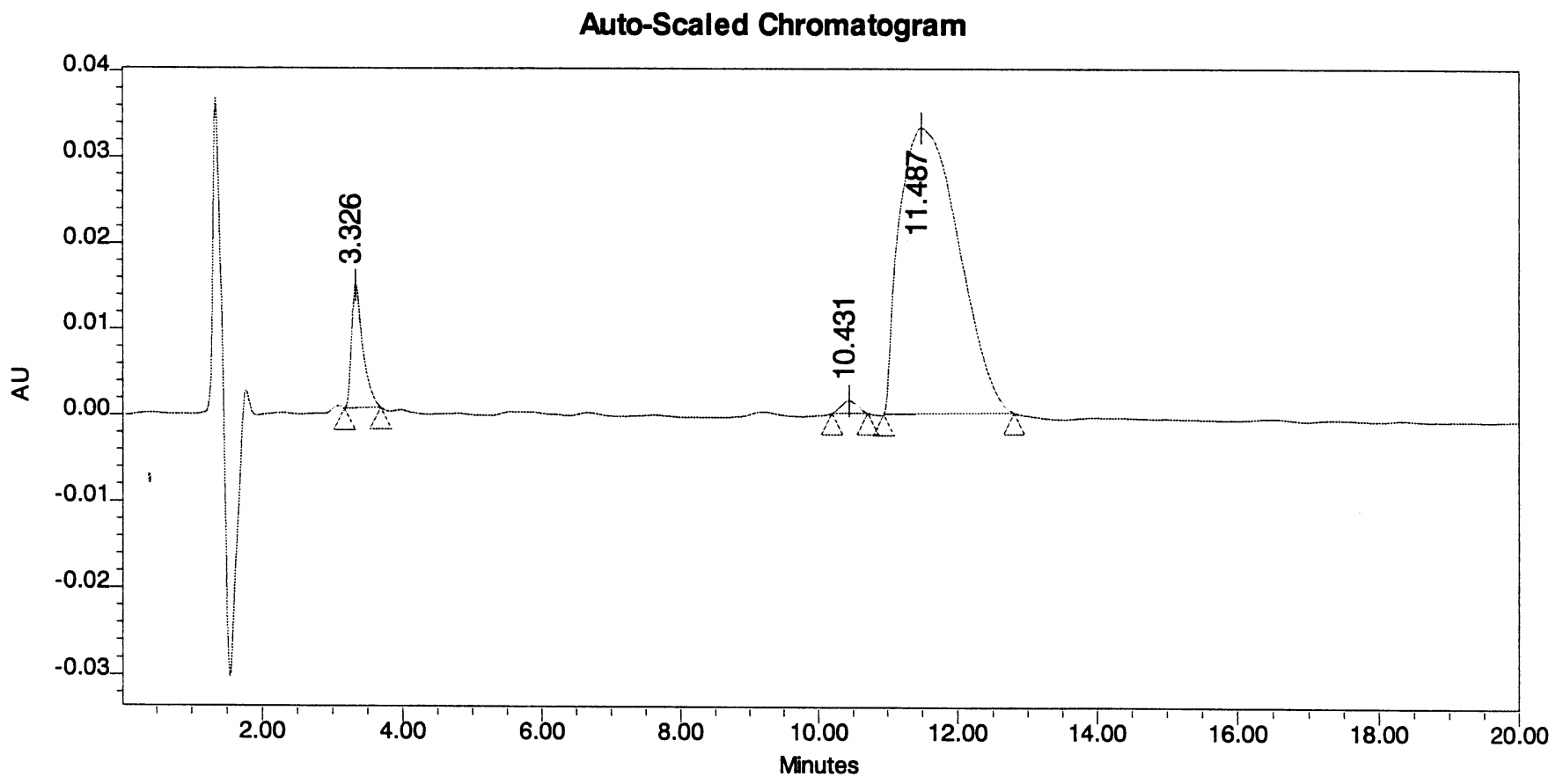

Peak Results

\begin{tabular}{|r|r|r|r|r|l|}
\hline & Name & RT & \multicolumn{1}{c|}{ Area } & \% Area & Peak Type \\
\hline 1 & & 3.326 & 162634 & 7.61 & Unknow n \\
\hline 2 & & 10.431 & 23806 & 1.11 & Unknow n \\
\hline 3 & & 11.487 & 1950561 & 91.28 & Unknow n \\
\hline
\end{tabular}




\section{Sample Information}

$$
\text { purealidin } A-2
$$

\author{
Sample Type Unknow n \\ Date Acquired 11/6/2005 10:57:14 AM \\ Acq Method Set fanglong
}

Processing Method LC Default Processing

Date Processed 11/7/2005 10:12:20 AM

Channel 2487Channel 1

Run Time $\quad 40.0$ Minutes

Auto-Scaled Chromatogram

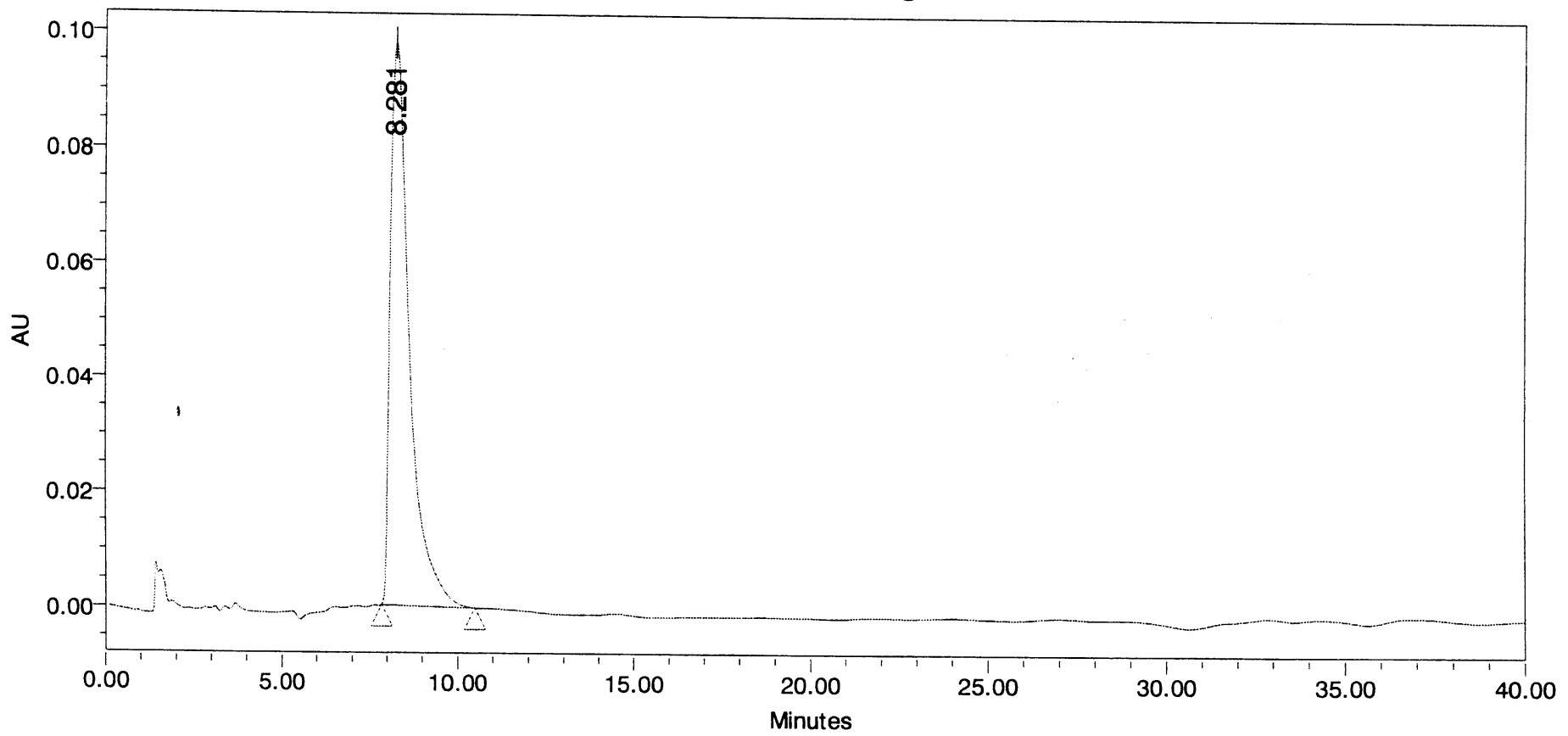

Peak Results

\begin{tabular}{|c|c|c|c|c|c|c|}
\hline & Name & RT & Area & Height & Amount & Units \\
\hline 1 & & 8.281 & 3743598 & 97832 & & \\
\hline
\end{tabular}

$$
\begin{aligned}
& \text { Condifions: } \\
& \text { Meor }=H_{20}=40=60 \\
& (0,1)(0 T F H) \\
& \because \text { Socratic } \\
& \text { Hon: nogl/min }
\end{aligned}
$$




\section{Sample Information}

$\begin{array}{llll}\text { SampleName } & \text { yfl-purealidin-8 } & \text { Sample Type } & \text { Unknown } \\ \text { Vial } & 1 & \text { Date Acquired } & \text { 11/6/2005 12:22:41 PM } \\ \text { Injection } & 1 & \text { Acq Method Set fanglong } \\ \text { Injection Volume } & 10.00 \mathrm{ul} & \text { Processing Method LC Default Pocessing } \\ \text { Channel } & \text { 2487Channel 1 } & \text { Date Processed 11/7/2005 10:10:29 AM } \\ \text { Run Time } & 40.0 \text { Minutes } & \end{array}$

Auto-Scaled Chromatogram

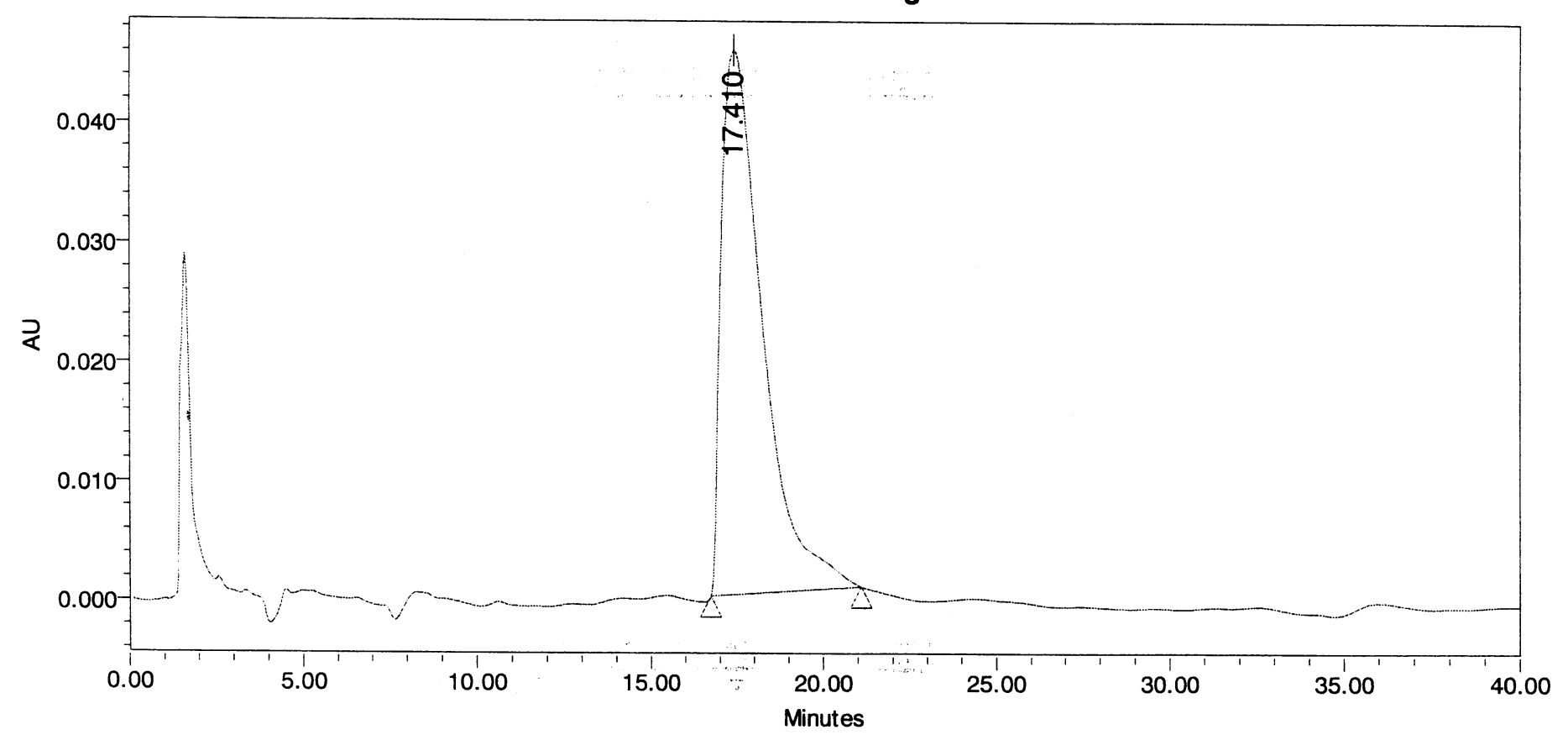

Peak Results

\begin{tabular}{|c|c|c|c|c|c|c|}
\hline & Name & RT & Area & Height & Amount & Units \\
\hline 1 & & 17.410 & 3812928 & 45691 & & \\
\hline
\end{tabular}

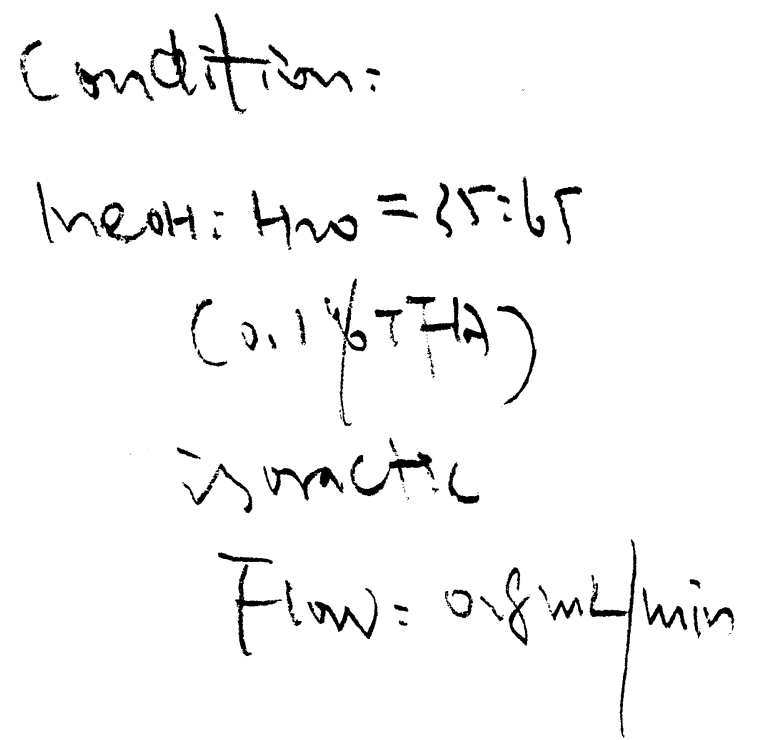




\section{Sample Information}

$\begin{array}{ll}\text { SampleName } & \text { yfl1a1b-30 }(43 a, a) \\ \text { Injection Volume } & 10.00 \text { ul } \\ \text { Channel } & \text { 2487Channel } 1 \\ \text { Run Time } & 20.0 \text { Minutes }\end{array}$

sample_info Enter Comment here!
Sample Type Unknown

Date Acquired $\quad$ 1/30/04 11:32:01 AM

Acq Method Set Generic_MS 2487

Processing Method christine

Date Processed 2/7/04 5:28:47 PM

Auto-Scaled Chromatogram

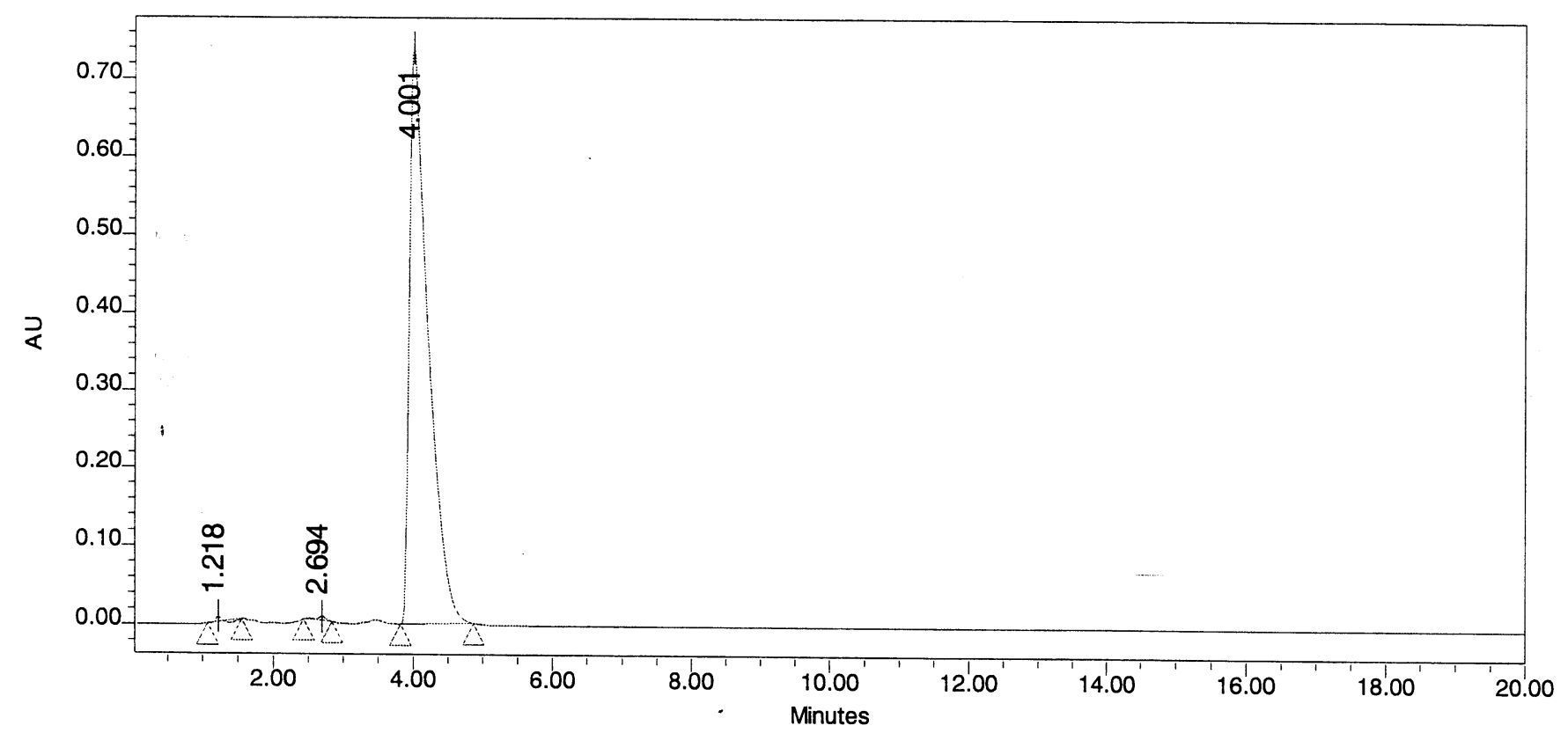

Peak Results

\begin{tabular}{|r|c|c|r|r|l|}
\hline & Name & RT & \multicolumn{1}{c|}{ Area } & \% Area & Peak Type \\
\hline 1 & & 1.218 & 59814 & 0.43 & Unknow $n$ \\
\hline 2 & & 2.694 & 47414 & 0.34 & Unknow $\mathrm{n}$ \\
\hline 3 & & 4.001 & 13767200 & 99.23 & Unknow $\mathrm{n}$ \\
\hline
\end{tabular}

canditais for 4346 comnow

$$
\begin{aligned}
& 65 \% \mathrm{H}_{2} \mathrm{O}, 35 \% \mathrm{CH} 3 \mathrm{CN} \\
& (0.1 \% T F A) \\
& \text { (isocratic) }
\end{aligned}
$$




\section{Sample Information}

$\begin{array}{ll}\text { SampleName } & 1 \mathrm{a} 2 \mathrm{~b}-3 \quad(4 \hat{\mathrm{a}}, \mathrm{b}) \\ \text { Injection Volume } & 10.00 \mathrm{ul} \\ \text { Channel } & 2487 \text { Channel } 1 \\ \text { Run Time } & \text { 20.0 Minutes }\end{array}$

sample_info Enter Comment here!
Sample Type Unknown

Date Acquired $\quad$ 1/26/04 10:18:50 PM

Acq Method Set Generic_MS 2487

Processing Method christine

Date Processed 1/28/04 11:04:07 AM

Auto-Scaled Chromatogram

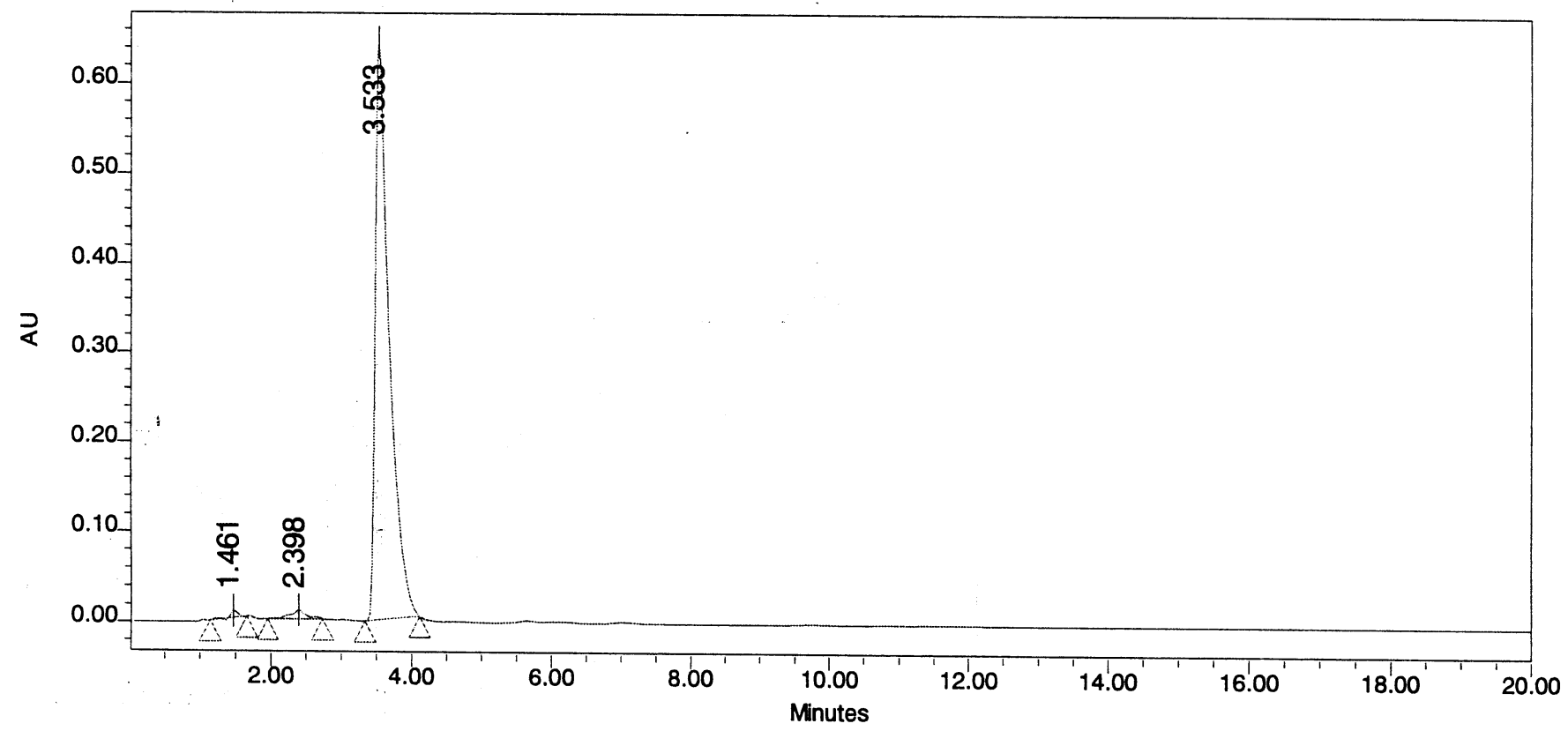

\begin{tabular}{|l|r|r|r|r|l|}
\hline \multicolumn{7}{|c|}{ Peak Results } \\
\hline & Name & RT & \multicolumn{1}{c|}{ Area } & $\%$ Area & Peak Type \\
\hline 1 & & 1.461 & 66911 & 0.73 & Unknow $n$ \\
\hline 2 & & 2.398 & 146916 & 1.60 & Unknow n \\
\hline 3 & & 3.533 & 8940825 & 97.66 & Unknow n \\
\hline
\end{tabular}




\section{Sample Information}

$\begin{array}{ll}\text { SampleName } & \text { 1a3b-3 } \quad(43 a, c) \\ \text { Injection Volume } & 10.00 \mathrm{ul} \\ \text { Channel } & \text { 2487Channel } 1 \\ \text { Run Time } & 20.0 \text { Minutes }\end{array}$

sample_info Enter Comment here!

\author{
Sample Type Unknown \\ Date Acquired 1/26/04 9:35:51 PM \\ Acq Method Set Generic_MS 2487 \\ Processing Method christine \\ Date Processed 1/28/04 11:01:31 AM
}

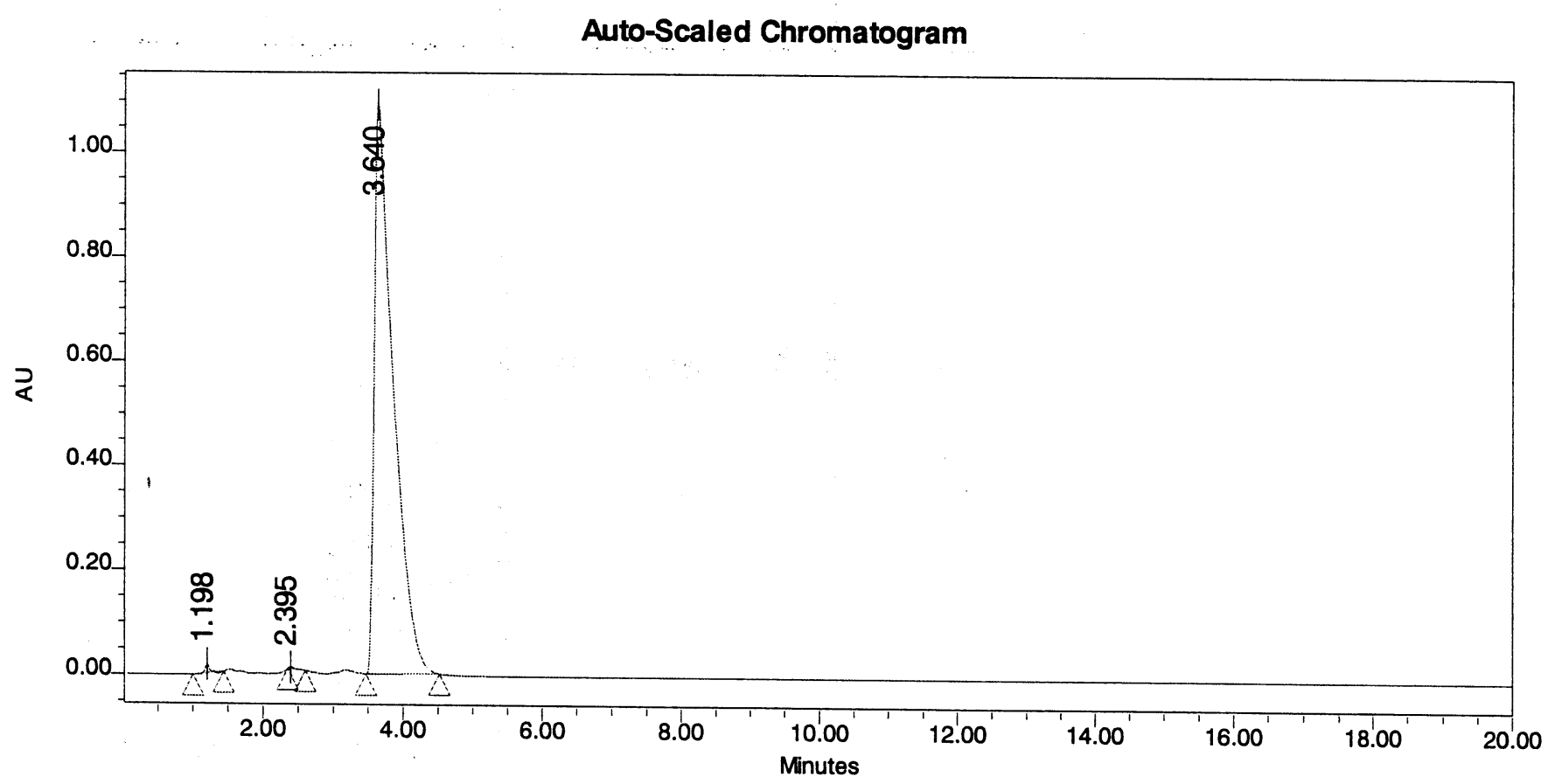

Peak Results

\begin{tabular}{|r|c|c|r|r|l|}
\hline & Name & RT & \multicolumn{1}{c|}{ Area } & \% Area & Peak Type \\
\hline 1 & & $1: 198$ & 80493 & 0.39 & Unknow $n$ \\
\hline 2 & & 2.395 & 21269 & 0.10 & Unknow $n$ \\
\hline 3 & & 3.640 & 20624979 & 99.51 & Unknow $n$ \\
\hline
\end{tabular}




\section{Sample Information}

$\begin{array}{ll}\text { SampleName } & 1 \mathrm{a} 4 \mathrm{~b}-3 \quad(43 a, d) \\ \text { Injection Volume } & 10.00 \mathrm{ul} \\ \text { Channel } & \text { 2487Channel 1 } \\ \text { Run Time } & \text { 20.0 Minutes }\end{array}$

Sample Type Unknown

Date Acquired 1/26/04 9:56:48 PM

Acq Method Set Generic_MS 2487

Processing Method christine

Date Processed 1/28/04 11:02:50 AM

sample_info Enter Comment here!

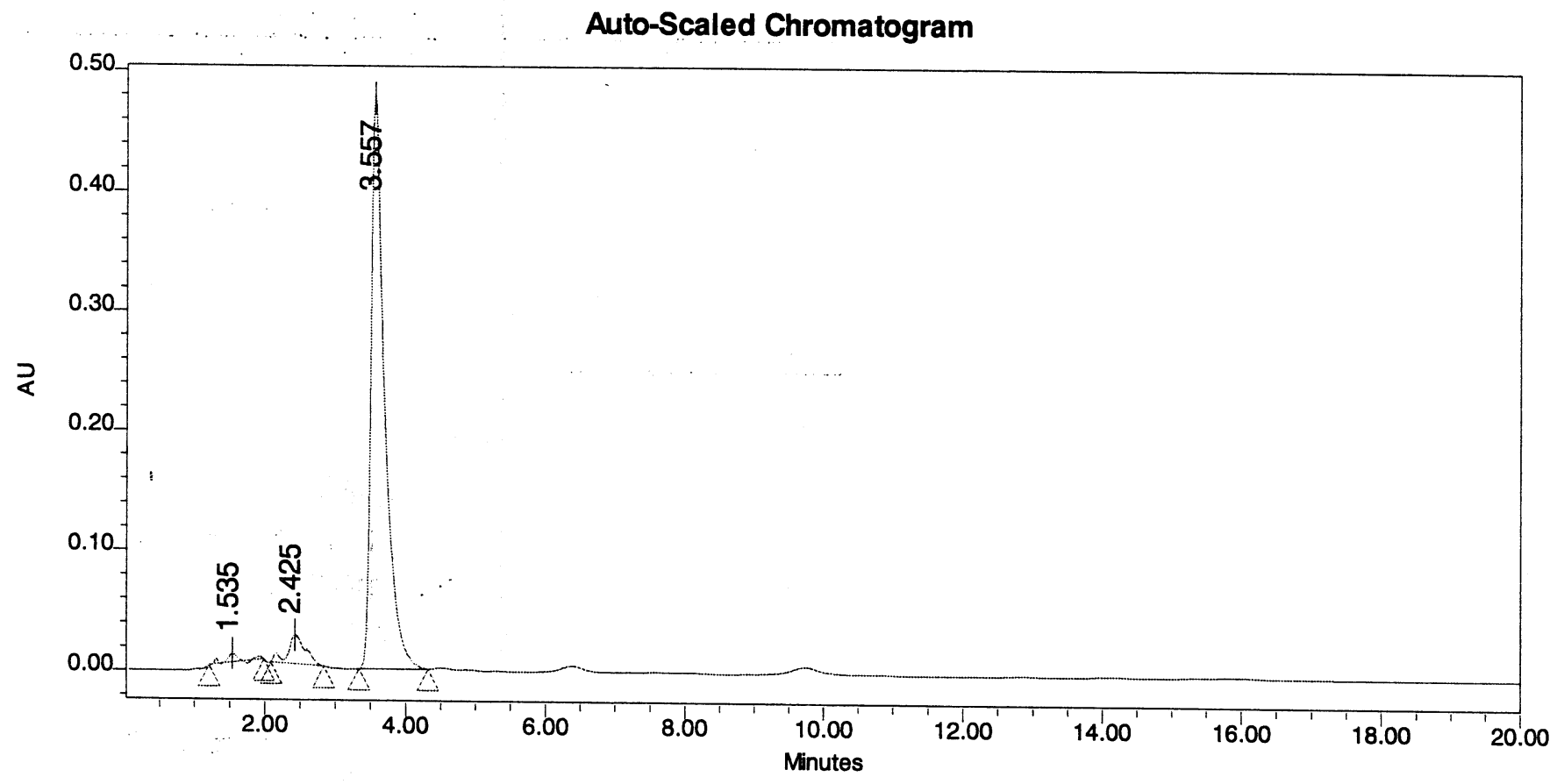

Peak Results

\begin{tabular}{|r|r|r|r|r|l|}
\hline & Name & RT & \multicolumn{1}{c|}{ Area } & \% Area & Peak Type \\
\hline 1 & & 1.535 & 106792 & 1.54 & Unknow $n$ \\
\hline 2 & & 2.425 & 421762 & 6.10 & Unknow n \\
\hline 3 & & 3.557 & 6384985 & 92.35 & Unknow $n$ \\
\hline
\end{tabular}




\section{Sample Information}

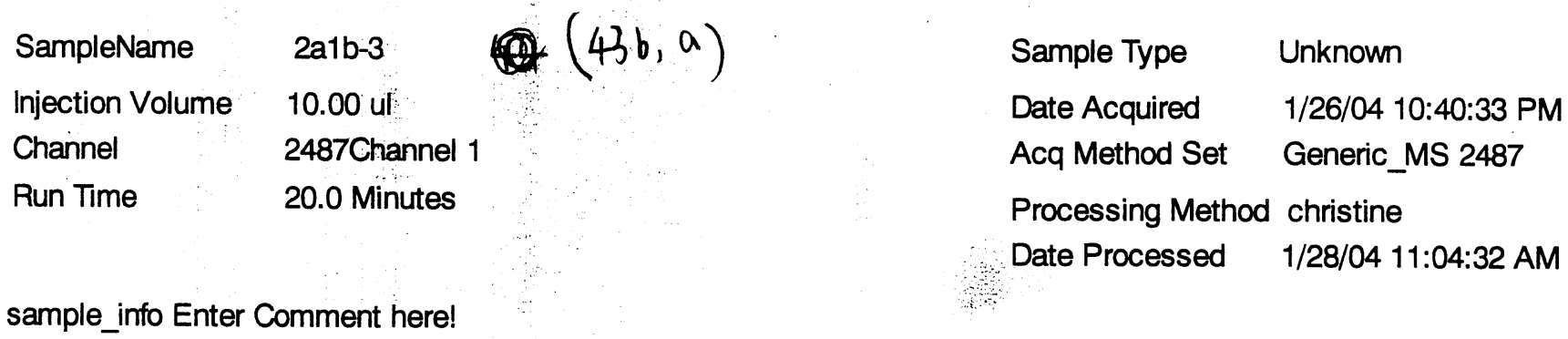

Auto-Scaled Chromatogram

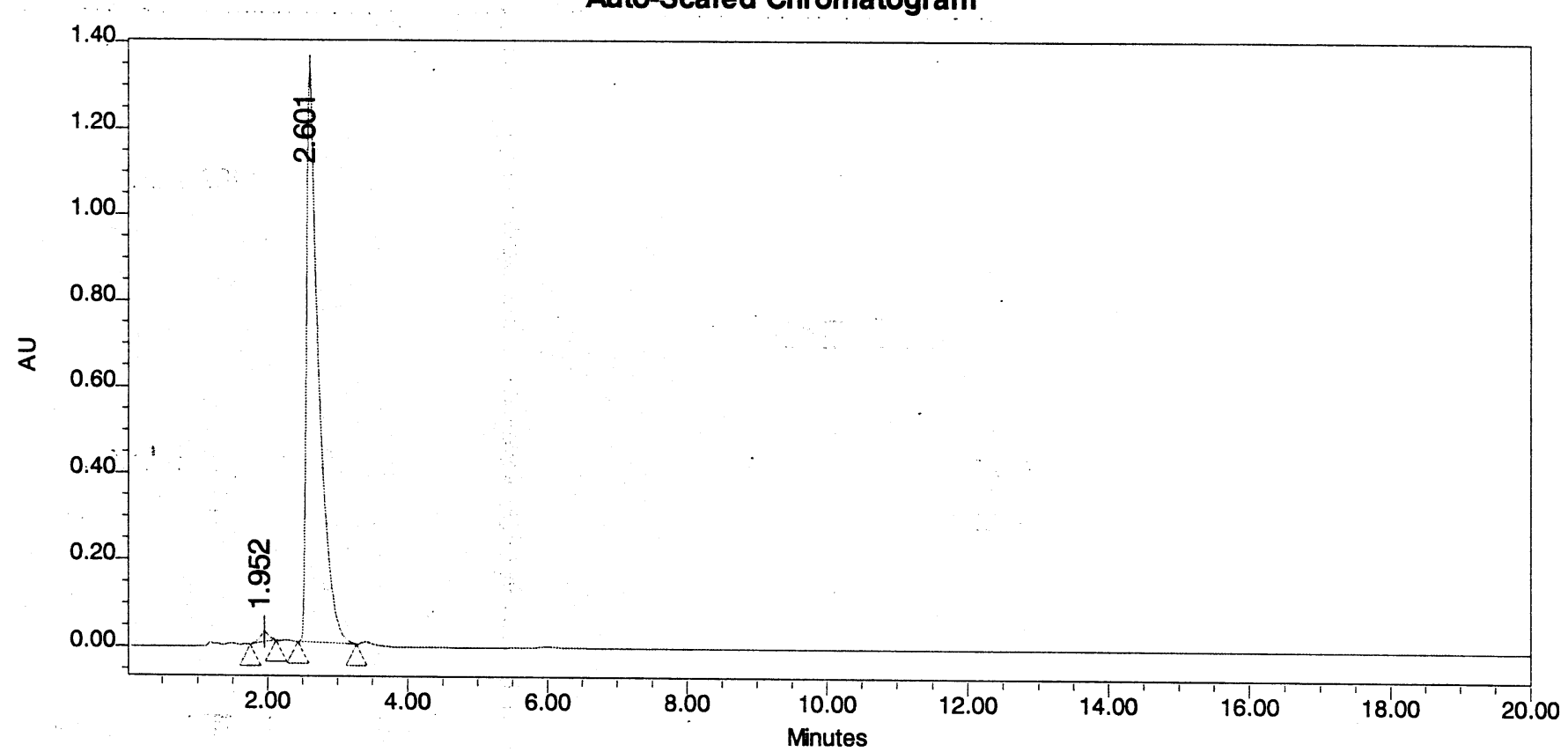

Peak Results

\begin{tabular}{|r|c|c|r|r|l|}
\hline & Name & RT & \multicolumn{1}{c|}{ Area } & \% Area & Peak Type \\
\hline 1 & & 1.952 & 200266 & 1.24 & Unknow n \\
\hline 2 & & 2.601 & 15915746 & 98.76 & Unknow n \\
\hline
\end{tabular}




\section{Sample Information}

$\begin{array}{ll}\text { SampleName } & \text { yfl2a2b-30 } \quad(43 b, b) \\ \text { Injection Volume } & 10.00 \mathrm{ul} \\ \text { Channel } & \text { 2487Channel } 1 \\ \text { Run Time } & \text { 20.0 Minutes }\end{array}$

Sample Type Unknown

Date Acquired 1/30/04 11:07:59 AM

Acq Method Set Generic_MS 2487

Processing Method christine

Date Processed 2/7/04 5:29:18 PM

sample_info Enter Comment here!

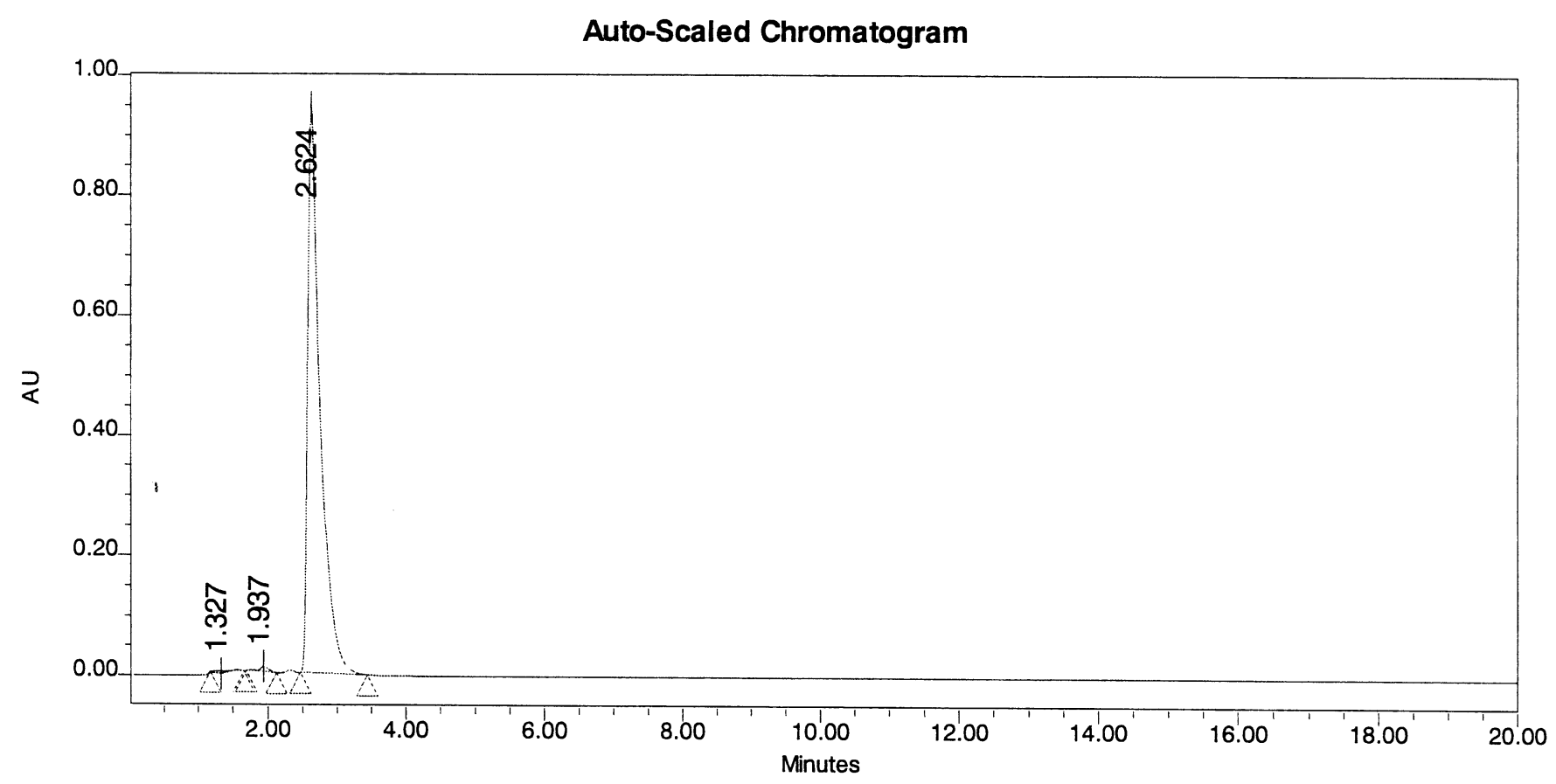

Peak Results

\begin{tabular}{|l|c|c|r|r|l|}
\hline & Name & RT & Area & \% Area & Peak Type \\
\hline 1 & & 1.327 & 51663 & 0.43 & Unknow $\mathrm{n}$ \\
\hline 2 & & 1.937 & 81302 & 0.68 & Unknow $\mathrm{n}$ \\
\hline 3 & & 2.624 & 11877767 & 98.89 & Unknow $\mathrm{n}$ \\
\hline
\end{tabular}




\section{Sample Information}

$\begin{array}{lll}\text { SampleName } & \text { yfl2a3b-30 } \quad(43 b, C) \\ \text { Injection Volume } & 10.00 \mathrm{ul} \\ \text { Channel } & \text { 2487Channel 1 } \\ \text { Run Time } & \text { 20.0 Minutes }\end{array}$

sample_info Enter Comment here!
Sample Type Unknown
Date Acquired $\quad$ 1/30/04 11:53:55 AM
Acq Method Set Generic_MS 2487
Processing Method christine
Date Processed 2/7/04 5:28:02 PM

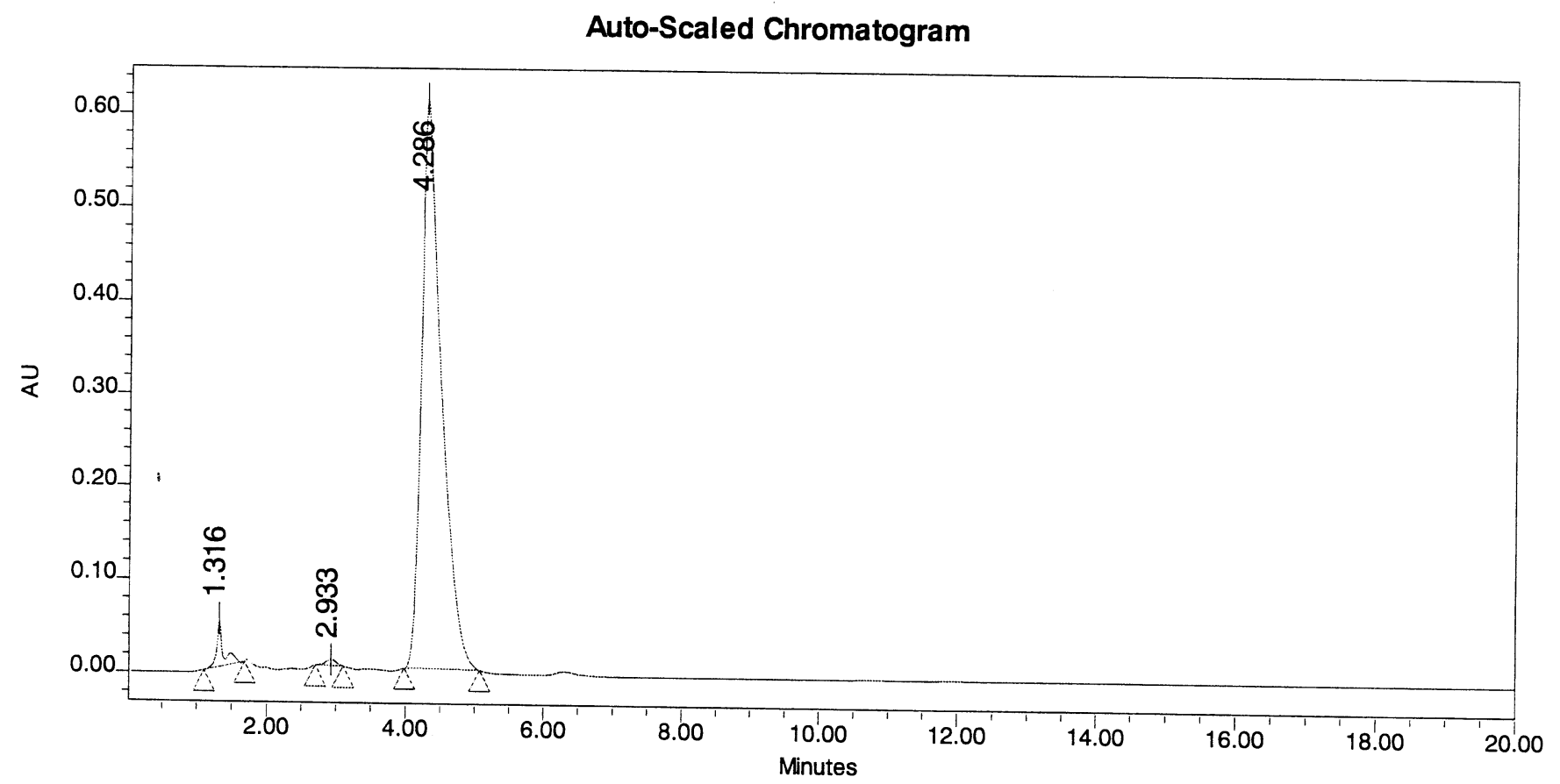

Peak Results

\begin{tabular}{|r|r|r|r|r|l|}
\hline & Name & RT & \multicolumn{1}{c|}{ Area } & \% Area & Peak Type \\
\hline 1 & & 1.316 & 331035 & 2.63 & Unknow $n$ \\
\hline 2 & & 2.933 & 70704 & 0.56 & Unknow $n$ \\
\hline 3 & & 4.286 & 12206600 & 96.81 & Unknow $n$ \\
\hline
\end{tabular}




\section{Sample Information}

$\begin{array}{llll}\text { SampleName } & \text { yfl-2a3b-5 }(436, C) & \text { Sample Type Unknow n } \\ \text { Vial } & 1 & \text { Date Acquired } & \text { 11/1/2005 5:51:22 PM } \\ \text { Injection } & 1 & \text { Acq Method Set fanglong } \\ \text { Injection Volume } & 10.00 \mathrm{ul} & \text { Processing Method LC Default Processing } \\ \text { Channel } & \text { 2487Channel 2 } & \text { Date Pocessed 11/4/2005 3:21:29 PM } \\ \text { Run Time } & \text { 40.0 Minutes } & \end{array}$

Auto-Scaled Chromatogram

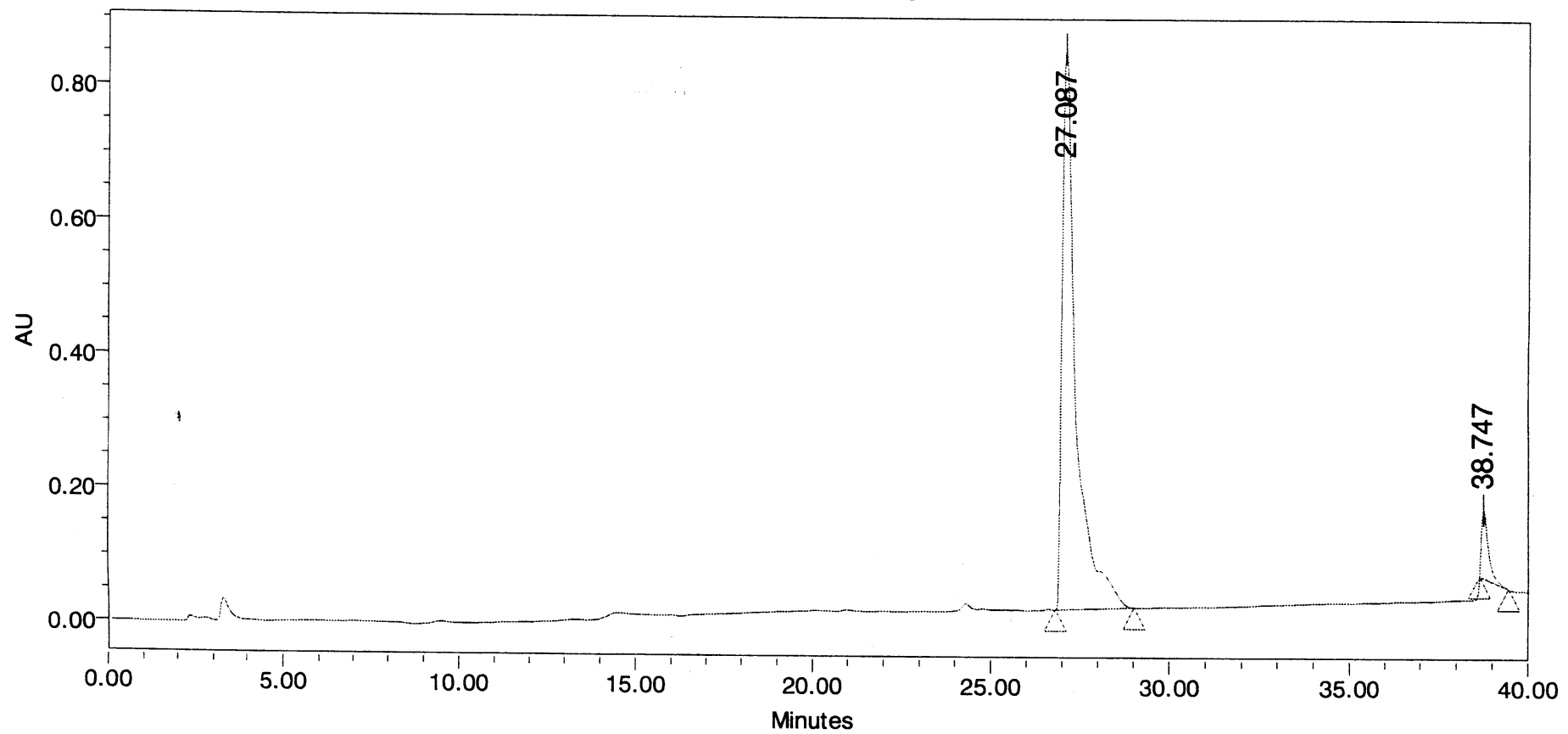

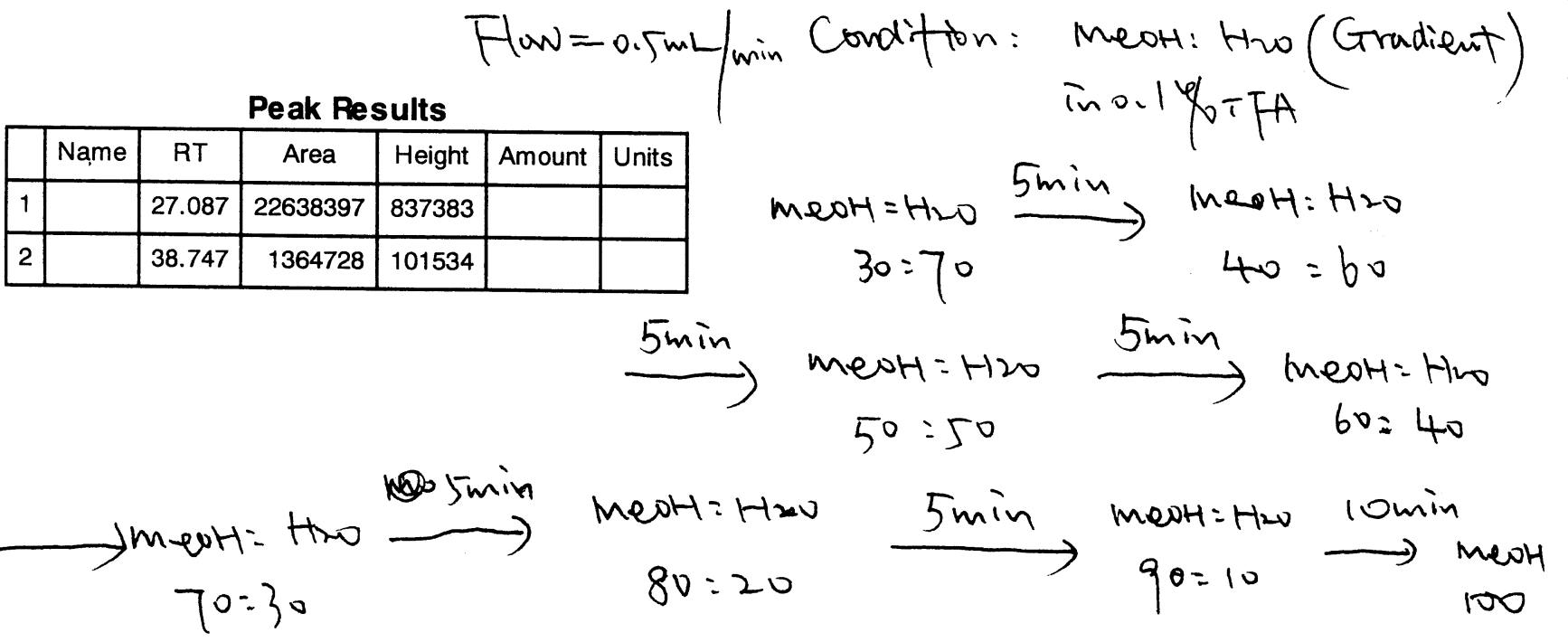




\section{Sample Information}

$\begin{array}{lll}\text { SampleName } & \text { 2a4b-3 } \quad(43 b, d) \\ \text { Injection Volume } & 10.00 \mathrm{ul} \\ \text { Channel } & \text { 2487Channel 1 } \\ \text { Run Time } & \text { 20.0 Minutes }\end{array}$

Sample Type Unknown

Date Acquired 1/26/04 11:01:26 PM

Acq Method Set Generic_MS 2487

Processing Method christine

Date Processed 1/28/04 11:05:06 AM

sample_info Enter Comment here!

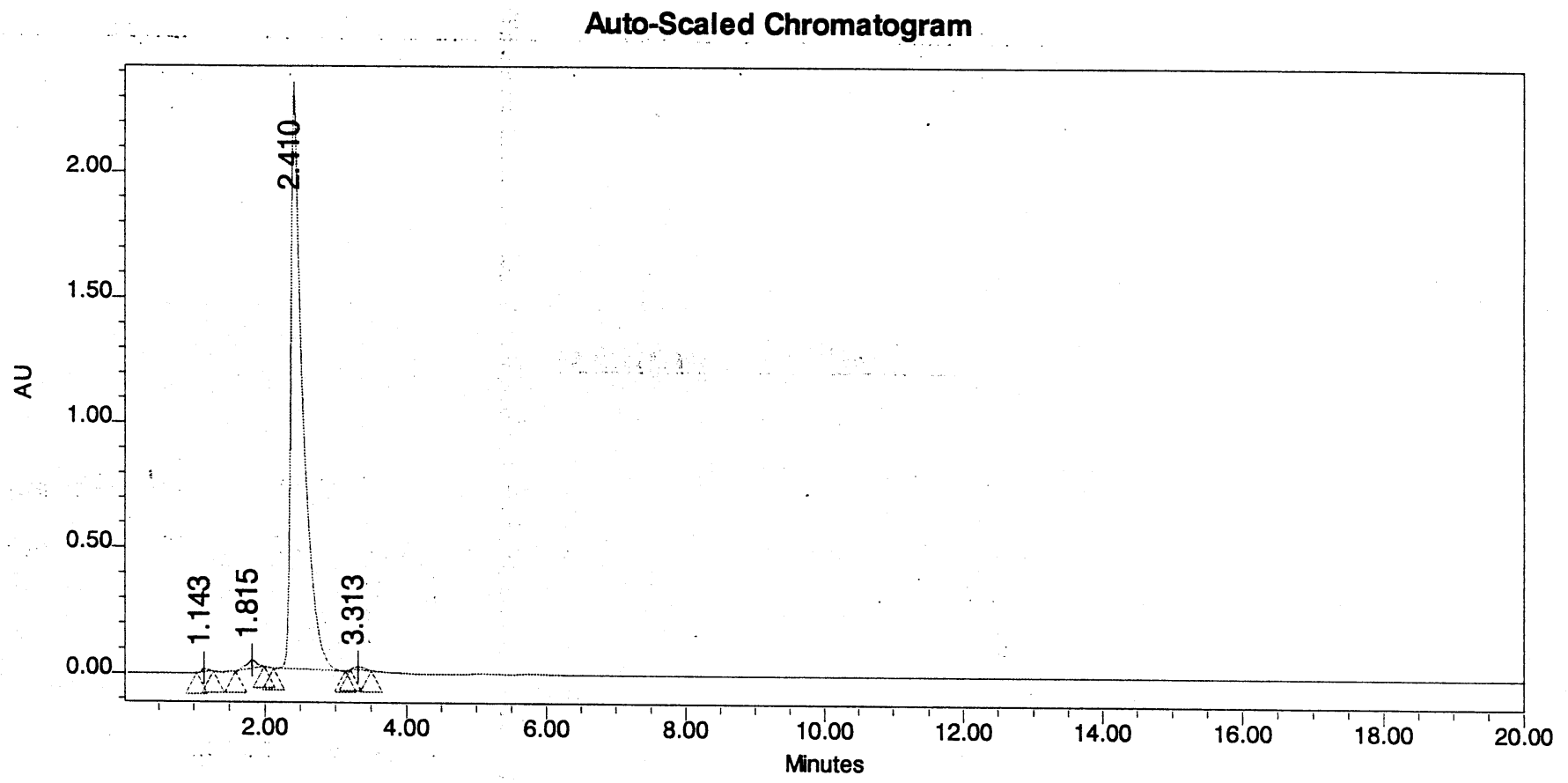

Peak Results

\begin{tabular}{|r|r|r|r|r|l|}
\hline & Name & RT & \multicolumn{1}{c|}{ Area } & \% Area & Peak Type \\
\hline 1 & & 1.143 & 77953 & 0.28 & Unknow n \\
\hline 2 & & 1.815 & 333856 & 1.20 & Unknow n \\
\hline 3 & & 2.410 & 27332060 & 98.04 & Unknow $n$ \\
\hline 4 & & 3.313 & 133567 & 0.48 & Unknow $n$ \\
\hline
\end{tabular}




\section{Sample Information}

$\begin{array}{ll}\text { SampleName } & 3 a 1 b-3 \quad(43 c, a) \\ \text { Injection Volume } & 10.00 \text { ul } \\ \text { Channel } & 2487 \text { Channel } 1 \\ \text { Run Time } & 20.0 \text { Minutes }\end{array}$

sample_info Enter Comment here!

\section{Sample Type Unknown \\ Date Acquired 1/26/04 6:26:27 PM \\ Acq Method Set Generic_MS 2487}

Processing Method christine

Date Processed 1/28/04 11:00:09 AM

Auto-Scaled Chromatogram

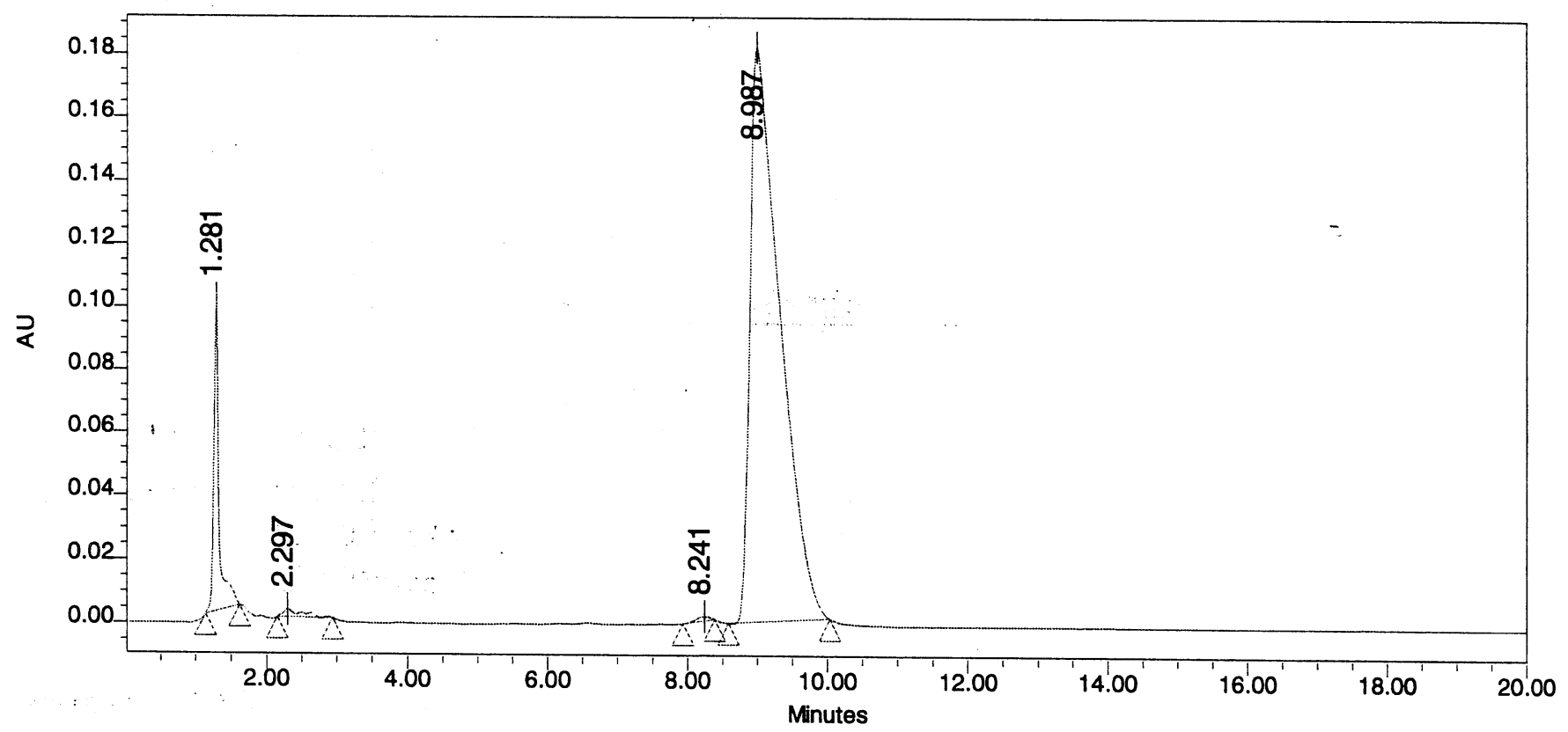

Peak Rēsuilts

\begin{tabular}{|r|c|c|r|r|l|}
\hline & Name & RT & \multicolumn{1}{c|}{ Area } & \% Area & Peak Type \\
\hline 1 & & 1.281 & 502647 & 8.45 & Unknow $n$ \\
\hline 2 & & 2.297 & 46155 & 0.78 & Unknow $n$ \\
\hline 3 & $\cdots$ & 8.241 & 20245 & 0.34 & Unknow $n$ \\
\hline 4 & & 8.987 & 5376109 & 90.43 & Unknow $n$ \\
\hline
\end{tabular}




\section{Sample Information}

$\begin{array}{ll}\text { SampleName } & 43 a 2 b-3 \quad(43 \mathrm{C}, \mathrm{b}) \\ \text { Injection Volume } & 10.00 \mathrm{ul} \\ \text { Channel } & \text { 2487Channel } 1 \\ \text { Run Time } & \text { 20.0 Minutes }\end{array}$

sample_info Enter Comment here!
Sample Type Unknown

Date Acquired $\quad$ 1/26/04 5:44:05 PM

Acq Method Set Generic_MS 2487

Processing Method christine

Date Processed 1/28/04 10:58:08 AM

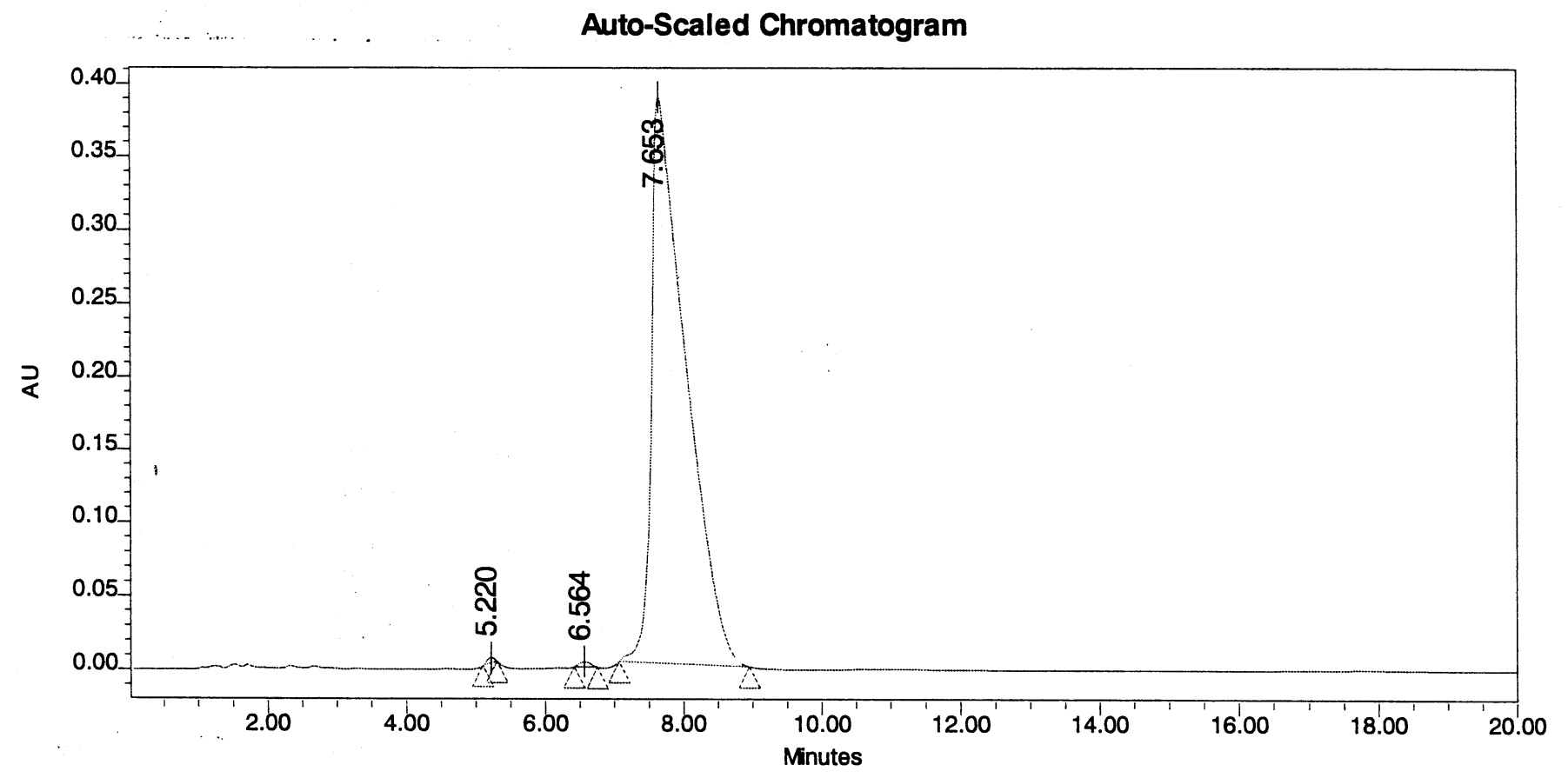

Peak Results

\begin{tabular}{|r|c|c|r|r|l|}
\hline & Name & RT & \multicolumn{1}{c|}{ Area } & \% Area & Peak Type \\
\hline 1 & & 5.220 & 26626 & 0.20 & Unknow $n$ \\
\hline 2 & & 6.564 & 40319 & 0.30 & Unknow $n$ \\
\hline 3 & & 7.653 & 13326002 & 99.50 & Unknow $n$ \\
\hline
\end{tabular}




\section{Sample Information}

$\begin{array}{ll}\text { SampleName } & 3 a 3 b-24-4 \\ \text { Injection Volume } & 10.00 \mathrm{ul} \\ \text { Channel } & \text { 2487Channel } 1 \\ \text { Run Time } & 50.0 \text { Minutes }\end{array}$

sample_info Enter Comment here!
Sample Type Unknown

Date Acquired $\quad$ 1/26/04 2:27:52 PM

Acq Method Set Generic_MS 2487

Processing Method christine

Date Processed 1/28/04 11:05:59 AM

Auto-Scaled Chromatogram

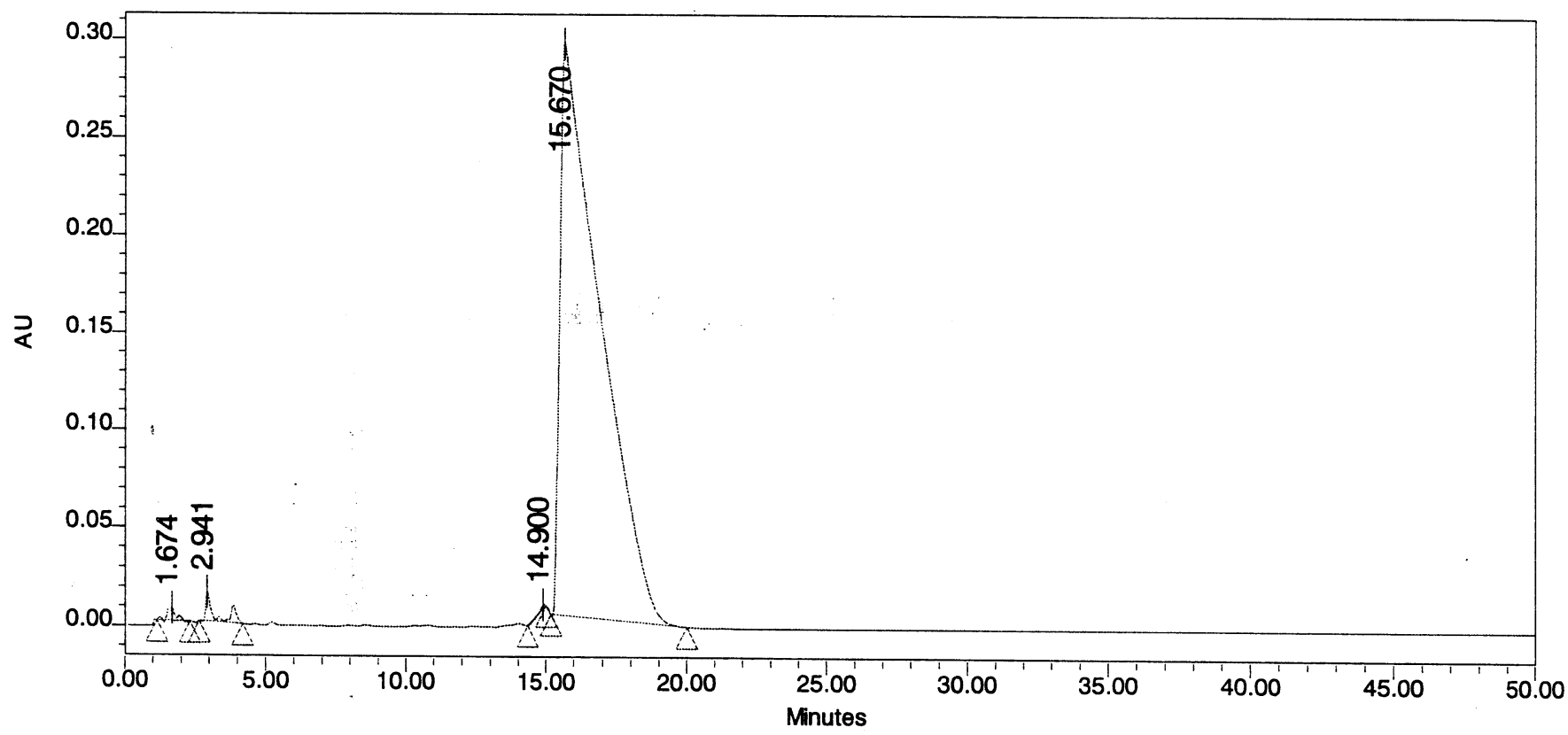

Peak Results

\begin{tabular}{|r|r|r|r|r|l|}
\hline & Name & RT & \multicolumn{1}{c|}{ Area } & \% Area & Peak Type \\
\hline 1 & & 1.674 & 140458 & 0.48 & Unknow $n$ \\
\hline 2 & & 2.941 & 309709 & 1.06 & Unknow $n$ \\
\hline 3 & & 14.900 & 34444 & 0.12 & Unknow $n$ \\
\hline 4 & & 15.670 & 28654214 & 98.34 & Unknow $n$ \\
\hline
\end{tabular}




\section{Sample Information}

$\begin{array}{lllll}\text { SampleName } & 3 a 4 b-3 & (43 C, d) & \text { Sample Type } & \text { Unknown } \\ \text { Injection Volume } & 10.00 \mathrm{ul} & & \text { Date Acquired } & \text { 1/26/04 6:05:42 PM } \\ \text { Channel } & 2487 \text { Channel 1 } & \text { Acq Method Set } & \text { Generic_MS 2487 } \\ \text { Run Time } & 20.0 \text { Minutes } & \text { Processing Method christine } \\ & & \text { Date Processed 1/28/04 10:58:37 AM } \\ & & \end{array}$

Auto-Scaled Chromatogram

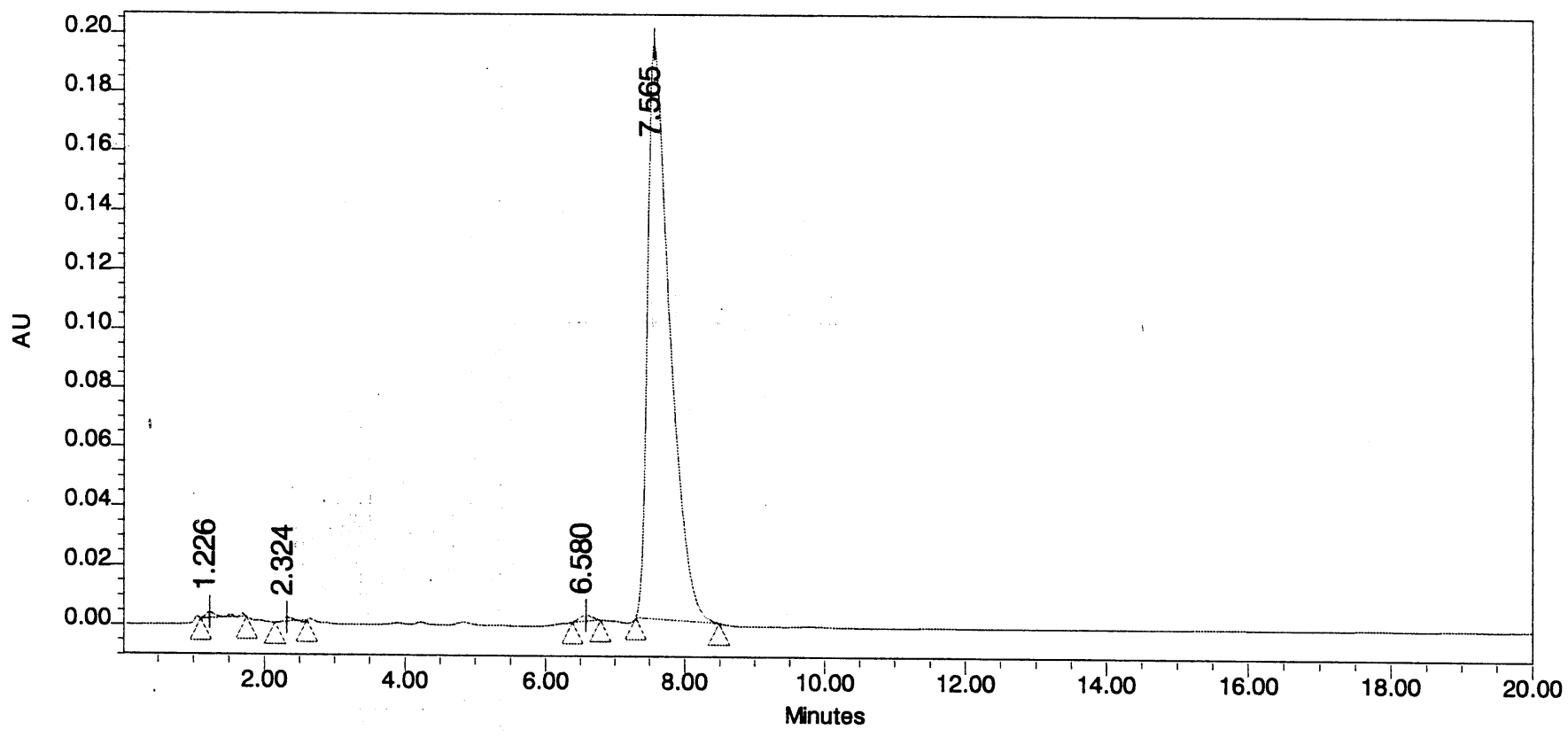

Peak Results

\begin{tabular}{|l|r|r|r|r|l|}
\hline & Name & RT & \multicolumn{1}{|c|}{ Area } & \% Area & Peak Type \\
\hline 1 & & 1.226 & 27927 & 0.64 & Unknow $n$ \\
\hline 2 & & 2.324 & 13802 & 0.32 & Unknow $n$ \\
\hline 3 & & 6.580 & 24973 & 0.58 & Unknow $n$ \\
\hline 4 & & 7.565 & 4269256 & 98.46 & Unknow $n$ \\
\hline
\end{tabular}


Sample Information

$\begin{array}{llll}\text { SampleName } & 4 a 1 b-3 \quad(43 d, a) & \text { Sample Type } & \text { Unknown } \\ \text { Injection Volume } & 10.00 \mathrm{ul} & \text { Date Acquired } & \text { 1/26/04 4:13:53 PM } \\ \text { Channel } & \text { 2487Channel 1 } & \text { Acq Method Set Generic_MS 2487 } \\ \text { Run Time } & \text { 25.0 Minutes } & \text { Processing Method christine } \\ & & \text { Date Processed } & \text { 1/28/04 10:55:54 AM } \\ \text { sample_info Enter Comment here! } & \end{array}$

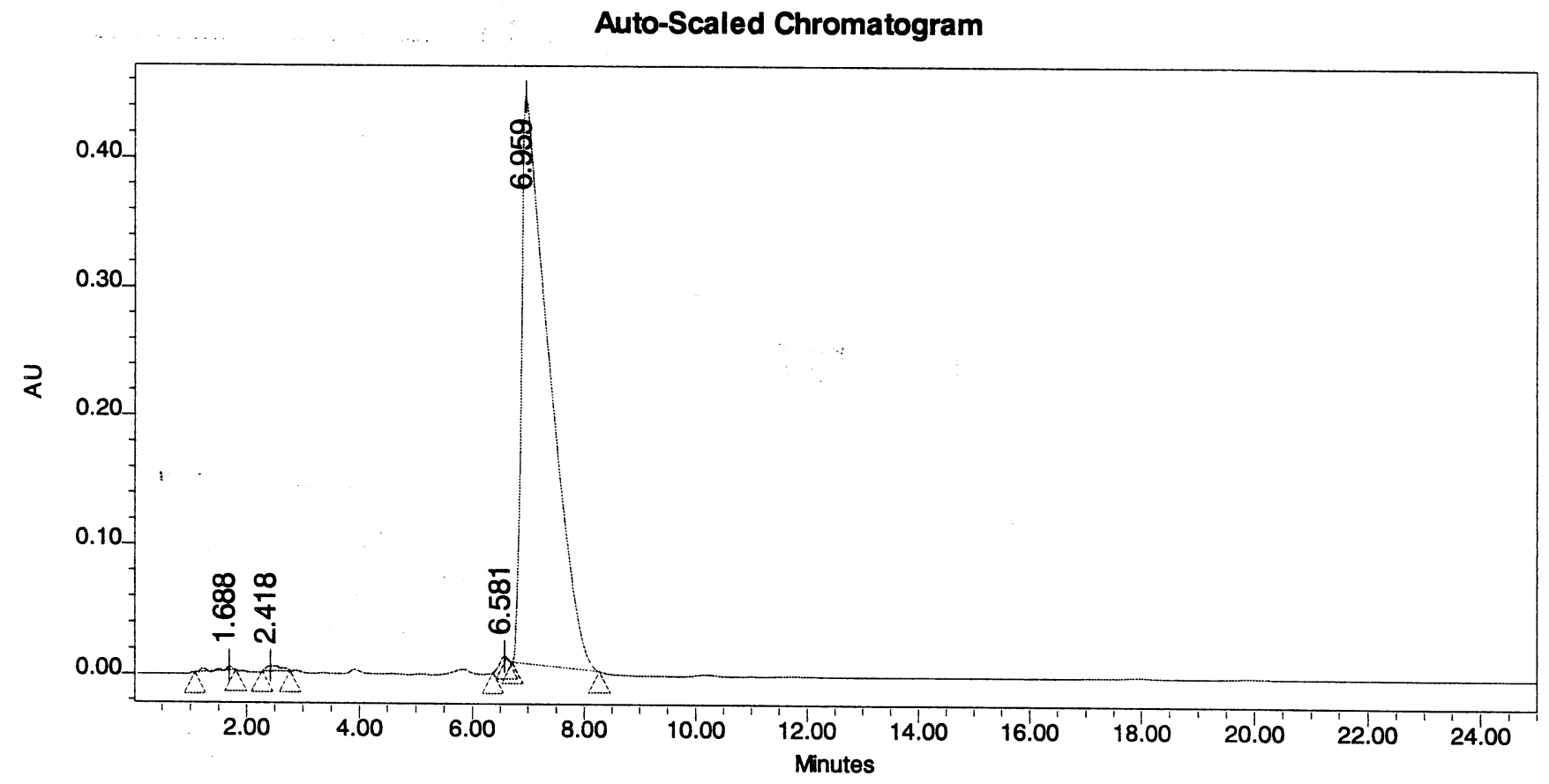

Peak Results

\begin{tabular}{|l|r|r|r|r|l|}
\hline & Name & RT & \multicolumn{1}{c|}{ Area } & \% Area & Poak Type \\
\hline 1 & & 1.688 & 49409 & 0.32 & Unknow n \\
\hline 2 & & 2.418 & 69434 & 0.45 & Unknow $n$ \\
\hline 3 & & 6.581 & 22037 & 0.14 & Unknow $n$ \\
\hline 4 & & 6.959 & 15140077 & 99.08 & Unknow $n$ \\
\hline
\end{tabular}




\section{Sample Information}

$\begin{array}{llll}\text { SampleName } & 4 a 2 b-3 \quad(43 d, b) & \text { Sample Type } & \text { Unknown } \\ \text { Injection Volume } & 10.00 \mathrm{ul} & \text { Date Acquired } & \text { 1/26/04 5:01:25 PM } \\ \text { Channel } & \text { 2487Channel 1 } & \text { Acq Method Set } & \text { Generic_MS 2487 } \\ \text { Run Time } & \text { 20.0 Minutes } & \text { Processing Method christine } \\ & & \text { Date Processed } & \text { 1/28/04 10:56:55 AM } \\ & & \end{array}$

\section{Auto-Scaled Chromatogram}

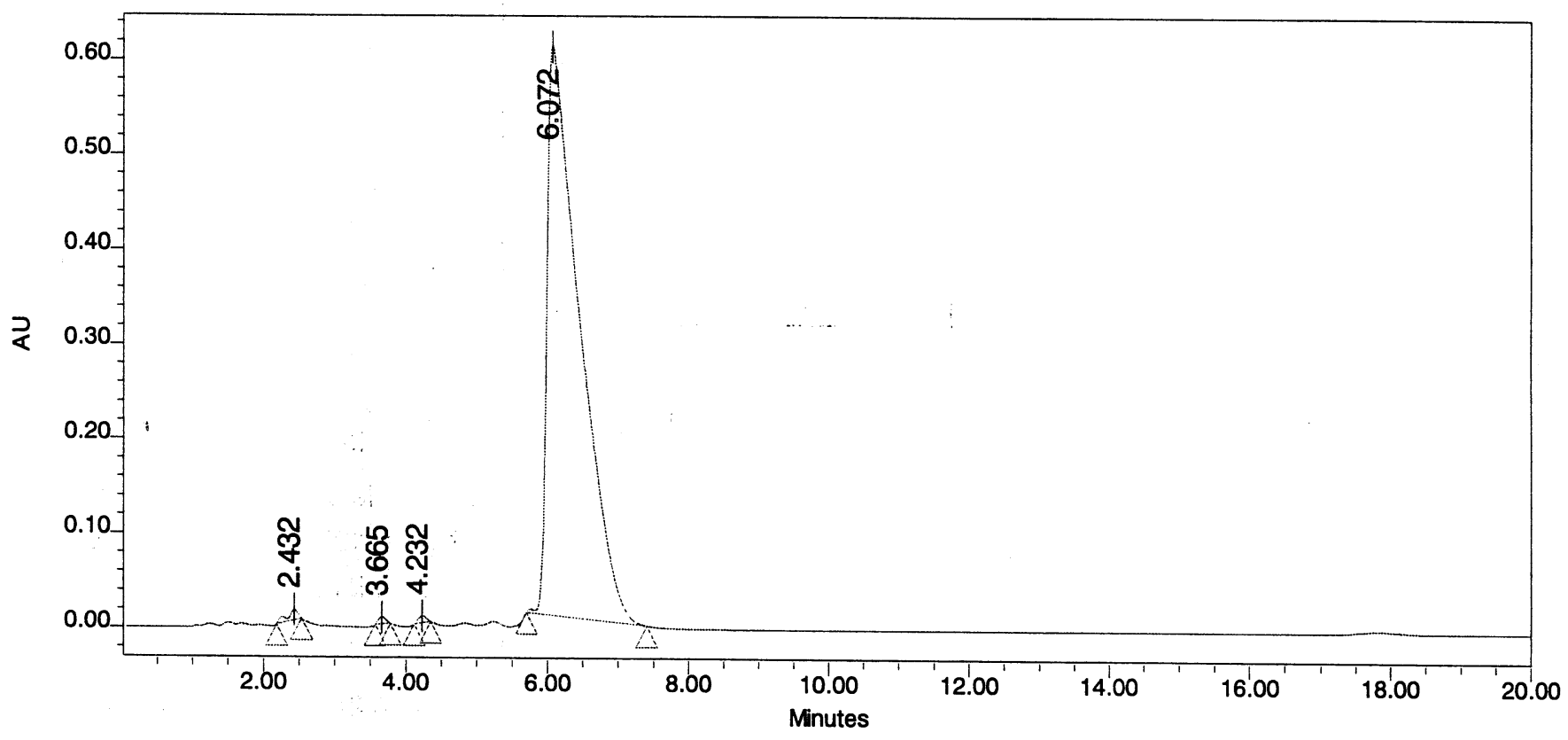

Peak Results

\begin{tabular}{|l|r|r|r|r|l|}
\hline & Name & \multicolumn{1}{|c|}{ RT } & \multicolumn{1}{c|}{ Area } & \% Area & Peak Type \\
\hline 1 & & 2.432 & 112405 & 0.58 & Unknow $n$ \\
\hline 2 & & 3.665 & 52844 & 0.27 & Unknow $n$ \\
\hline 3 & & 4.232 & 58603 & 0.30 & Unknow $n$ \\
\hline 4 & & 6.072 & 19277680 & 98.85 & Unknow $n$ \\
\hline
\end{tabular}




\section{Sample Information}

$\begin{array}{ll}\text { SampleName } & \text { 4a3b-3 } \quad(43 d, C) \\ \text { Injection Volume } & 10.00 \mathrm{ul} \\ \text { Channel } & \text { 2487Channel } 1 \\ \text { Run Time } & \text { 20.0 Minutes }\end{array}$

Sample Type Unknown

Date Acquired $\quad$ 1/26/04 4:40:16 PM

Acq Method Set Generic_MS 2487

Processing Method christine

Date Processed 1/28/04 10:56:28 AM

sample_info Enter Comment here!

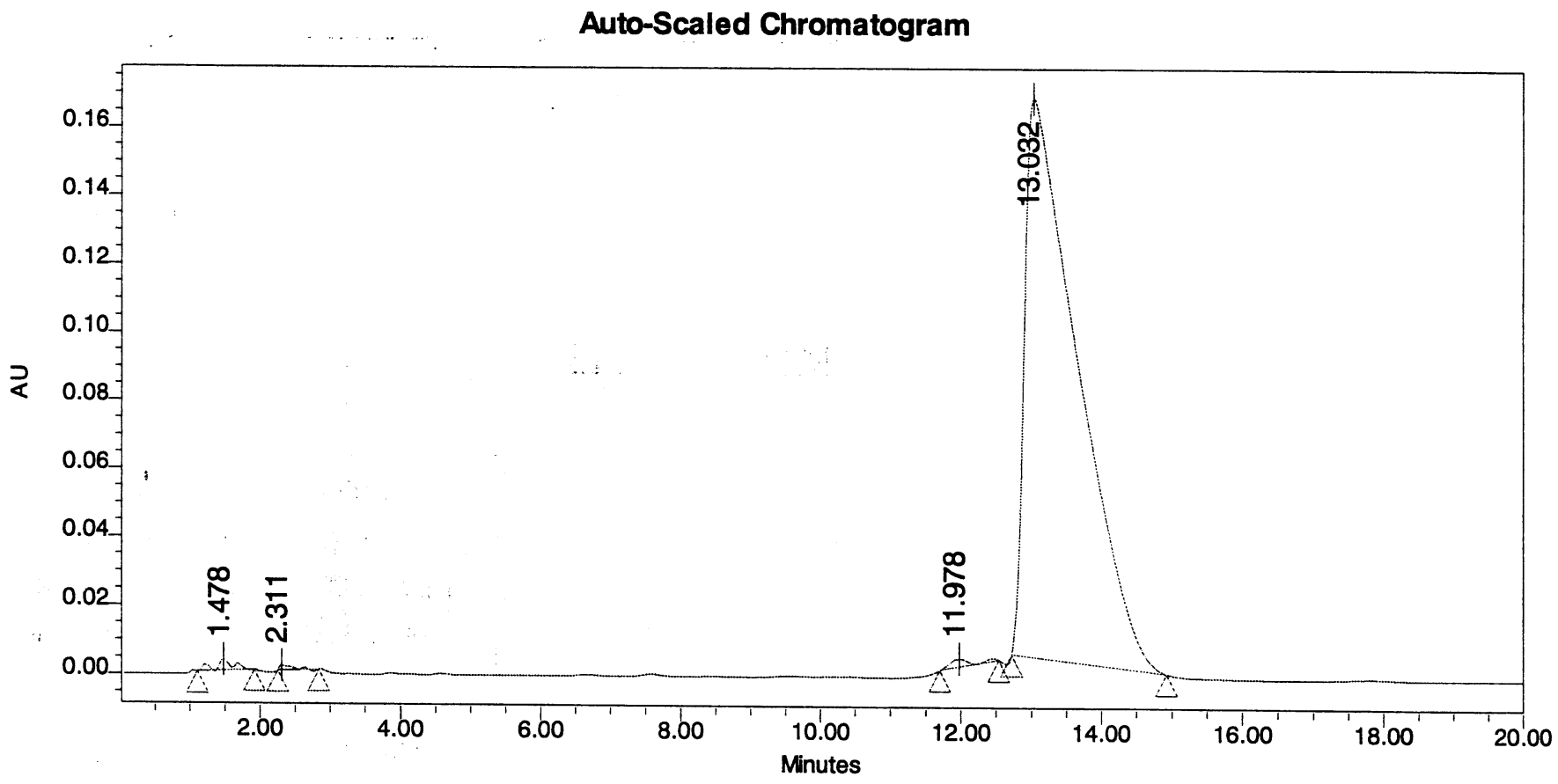

Peak Results

\begin{tabular}{|r|r|r|r|r|l|}
\hline & Name & RT & \multicolumn{1}{c|}{ Area } & \% Area & Peak Type \\
\hline 1 & & 1.478 & 53286 & 0.61 & Unknow $n$ \\
\hline 2 & & 2.311 & 19892 & 0.23 & Unknow $n$ \\
\hline 3 & & 11.978 & 48238 & 0.55 & Unknow $n$ \\
\hline 4 & & 13.032 & 8580058 & 98.60 & Unknow $n$ \\
\hline
\end{tabular}




\section{Sample Information}

$\begin{array}{ll}\text { SampleName } & 4 \mathrm{a} 4 \mathrm{~b}-3 \\ \text { Injection Volume } & 10.00 \mathrm{ul}\end{array} \quad(43 d, d)$

sample_info Enter Comment here!
Sample Type Unknown

Date Acquired 1/26/04 5:22:46 PM

Acq Method Set Generic_MS 2487

Processing Method christine

Date Processed 1/28/04 10:57:28 AM

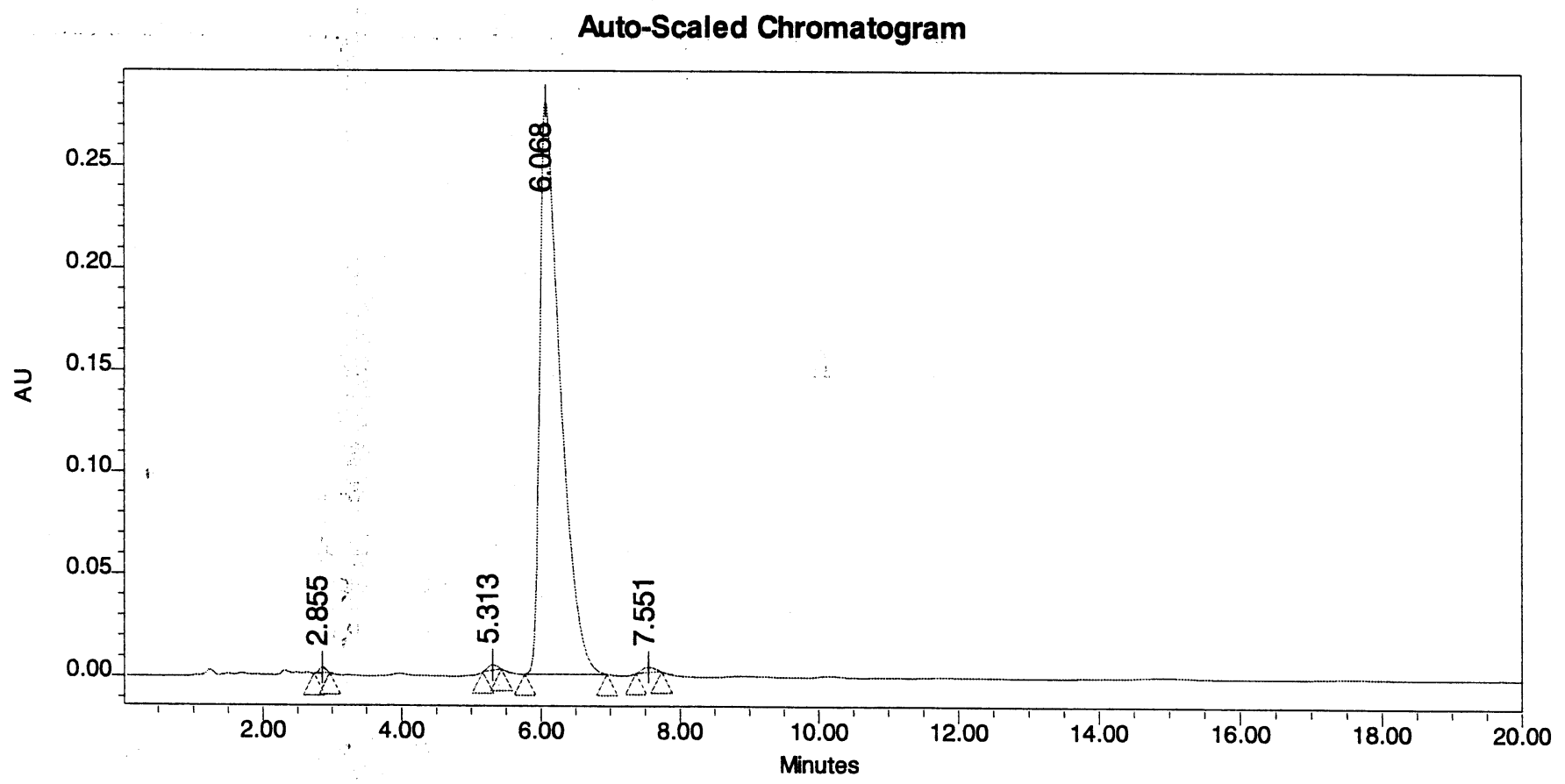

Peak Rèsults

\begin{tabular}{|r|r|r|r|r|l|}
\hline & Name & RT & \multicolumn{1}{c|}{ Area } & \% Area & Peak Type \\
\hline 1 & & 2.855 & 18632 & 0.32 & Unknow n \\
\hline 2 & & 5.313 & 25645 & 0.44 & Unknow n \\
\hline 3 & & 6.068 & 5727633 & 98.65 & Unknow n \\
\hline 4 & & 7.551 & 34260 & 0.59 & Unknow $n$ \\
\hline
\end{tabular}

